This item was submitted to Loughborough's Research Repository by the author.

Items in Figshare are protected by copyright, with all rights reserved, unless otherwise indicated.

\title{
Spatial transmission models: A taxonomy and framework
}

PLEASE CITE THE PUBLISHED VERSION

https://doi.org/10.1111/risa.13142

PUBLISHER

Wiley @ Society for Risk Analysis

VERSION

AM (Accepted Manuscript)

PUBLISHER STATEMENT

This is the peer reviewed version of the following article: ROBERTSON, D.A., 2018. Spatial transmission models: A taxonomy and framework. Risk Analysis, 39 (1), pp.225-243. which has been published in final form at https://doi.org/10.1111/risa.13142. This article may be used for non-commercial purposes in accordance with Wiley Terms and Conditions for Use of Self-Archived Versions

\section{LICENCE}

CC BY-NC-ND 4.0

\section{REPOSITORY RECORD}

Robertson, Duncan. 2018. “Spatial Transmission Models: A Taxonomy and Framework”. figshare. https://hdl.handle.net/2134/33296. 


\title{
SPATIAL TRANSMISSION MODELS: A TAXONOMY AND FRAMEWORK
}

\author{
To appear in Risk Analysis \\ Duncan A. Robertson \\ School of Business and Economics, Loughborough University, LEII 3TU, United Kingdom \\ Please cite as: \\ Robertson, D. A. (2018, In Press) 'Spatial Transmission Models: A Taxonomy and Framework', Risk Analysis
}




\begin{abstract}
Within risk analysis and more broadly, the decision behind the choice of which modelling technique to use to study the spread of disease, epidemics, fires, technology, rumors, or more generally spatial dynamics, is not well documented.
\end{abstract}

While individual models are well defined and the modeling techniques are well understood by practitioners, there is little deliberate choice made as to the type of model to be used, with modelers using techniques that are well accepted in the field, sometimes with little thought as to whether alternative modelling techniques could or should be used.

In this paper, we divide modelling techniques for spatial transmission into four main categories: population-level models, where a macro-level estimate of the infected population is required; cellular models, where the transmission takes place between connected domains, but is restricted to a fixed topology of neighboring cells; network models, where host-to-host transmission routes are modelled, either as planar spatial graphs or where short cuts can take place as in social networks; and finally agent-based models which model the local transmission between agents, either as host-to-host geographical contacts, or by modelling the movement of the disease vector, with dynamic movement of hosts and vectors possible, on a Euclidian space or a more complex space deformed by the existence of information about the topology of the landscape using GIS techniques.

We summarize these techniques by introducing a taxonomy classifying these modeling approaches. Finally, we present a framework for choosing the most appropriate spatial modelling method, highlighting the links between seemingly disparate methodologies, bearing in mind that the choice of technique rests with the subject expert.

\title{
KEYWORDS
}

Spatial models, transmission, model comparison 


\section{INTRODUCTION}

Spatial transmission is widely studied, particularly in the field of epidemiology (Riley 2007, Pfeiffer et al. 2008), but also in fields as diverse as connectivity on wireless networks (Andrews et al. 2010), the spread of fire (Rothermel 1972), marketing (Bradlow et al. 2005), and the diffusion of technology (Berger 2001). Risk analysis models which model the transmission of threats around a system include analytical models for epidemiological studies (Eisenberg et al. 1996, Moreno and Alvar 2002, Zagmutt et al. 2016), cellular automata models for nuclear terrorism (Atkinson et al. 2008), soil contamination (Cox 1999) and species invasion (Sikder et al. 2006), domain based studies of exposure to ozone (Fann et al. 2012), network models for communication (Dettmann and Georgiou 2016), power networks (Zio and Sansavini 2011), and in the social amplification of risk (Kasperson et al. 1988).

However, little work has been done on how to decide on the appropriate modeling technique to use for a specific problem (Brennan et al. 2006) let alone the decision as to which modelling technique to use for risks associated with spatially-transmitted phenomena: the model choice is often not deliberate, but can be a subliminal process brought about by the modeler's familiarity with a particular modelling technique. Alternative modelling techniques that could give additional or alternative insights may not be considered, leading to a model that may not be the most appropriate choice for the problem domain being studied.

Inspired by the Riley et al. (2015) call to arms setting out challenges for spatial epidemic models, we set out in this paper a taxonomy for modelling spatial transmission into four broad categories: population-level models, cellular models, network models, and finally agent-based models.

Section 2 of this paper introduces Population Models which model the population, or proportions of the population that are infected or not, these being among the oldest techniques used in epidemiological modelling (Morabia 2004). They are however inherently non-spatial as the population as a whole is modelled, the dynamics coming from the interaction between, for example, susceptible and infected sub-populations. It is possible to make these models spatial by breaking 
down the populations into spatial domains, and introducing dynamics of the transfer between these spatial domains on top of the transitions between susceptible and infected states. Section 3 introduces Cellular Models that can be used to produce a representation of a space. Simple squares or hexagons, or in more sophisticated analyses, irregular shaped domains are used, where transmission occurs across domain boundaries. We then progress in section 4 to Network Models which identify the relationships between nodes forming a network. These relationships may represent transmission pathways as edges in a network model. Network models are represented as network graphs, and these graphs can be planar, in which case they can represent a Euclidian space such as the links between towns in a road network, or they may be non-planar, in which case 'short cuts' may be possible such as in a social network. And in section 5, Agent Models are an alternative methodology where both the movement of vectors and the movement of hosts can be incorporated into the model. We further introduce GIS (Geographic Information System) techniques that can be incorporated into a spatial model that can deform a regular space into a rich, non-uniform topology.

Throughout the paper, we emphasize the links between these techniques, and show that the representation in one technique may be replicated using an alternative modelling perspective. We set out in section 6 a taxonomy of modelling techniques, categorizing the differences between these techniques. Finally, in section 7, we set out a framework for assisting the decision as to which modelling technique should be used for risk analysis of spatially transmitted phenomena, emphasizing the links between seemingly disparate methods.

\section{POPULATION MODELS}

\subsection{SI/SIS/SIR Models}

Compartmental models such as SI, SIS, and SIR (Susceptible, Infected, Recovered) models are by far the most well studied transmission models, dating back to the work of Kermack and McKendrick (1927). The intuition behind these models is that the population is divided into two or more compartments. In the simplest form, the SI model, the compartments are one of two states, as a 
function of time, $t: S(t)$ (Susceptible) or $I(t)$ (Infected). A simple extension in the SIR model, is to include a third compartment, $R(t)$ (Recovered/Removed).

In their simplest form, the total population is considered fixed, with the proportion in each state varying over time, hence $S, I$, and $R$ being a function of time, $t$.

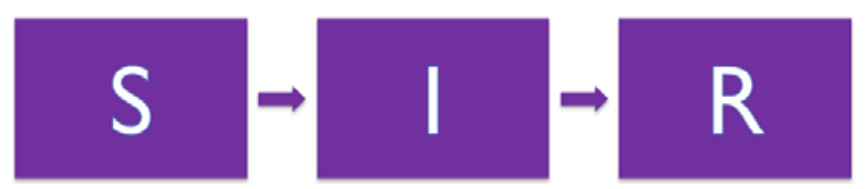

Figure 1: SIR Model Compartments

These models, which are a mainstay of the epidemiological risk community, use differential equations that link deterministically the populations in each state through linked differential equations (Hethcote 2000):

$$
\begin{array}{ll}
\frac{d S}{d t}=-\beta \frac{I S}{N} ; & S(0)=S_{0} \geq 0 ; \\
\frac{d I}{d t}=-\beta \frac{I S}{N}-\gamma I ; & I(0)=I_{0} \geq 0 ; \\
\frac{d R}{d t}=\gamma I ; & R(0)=R_{0} \geq 0
\end{array}
$$

where $S(t), I(t), R(t)$ are the numbers in the Susceptible, Infected, and Recovered states, such that $S(t)$ $+I(t)+R(t)=N$; with $\beta \& \gamma$ being constants.

The difference between application of the SIR model and the SIS model is that the SIR model is used for infectious diseases which confer immunity such as whooping cough or measles, whereas the SIS model is used for repeat infections such as influenza, gonorrhea or chlamydia.

Another extension is for the recovered state to be temporary, where after a delay, the recovered subpopulation becomes susceptible again, producing $S \rightarrow I \rightarrow R \rightarrow S$ transitions. 
In order to incorporate a spatial dimension into these models, subpopulations can be created where hosts move between patches at a certain rate, so the $S, I, R$ populations are further divided $S_{n}, I_{n}, R_{n}$ where $n$ is the index of the spatial region.

In the simplest form of a SI model, the familiar s-shaped dynamics are exhibited as shown in Figure 2, the logistic growth equation:

$$
\frac{d I}{d t}=\beta(1-I) I \quad ; \quad I(t)=\frac{I_{0} e^{\beta t}}{1-I_{0}+I_{0} e^{\beta t}}
$$

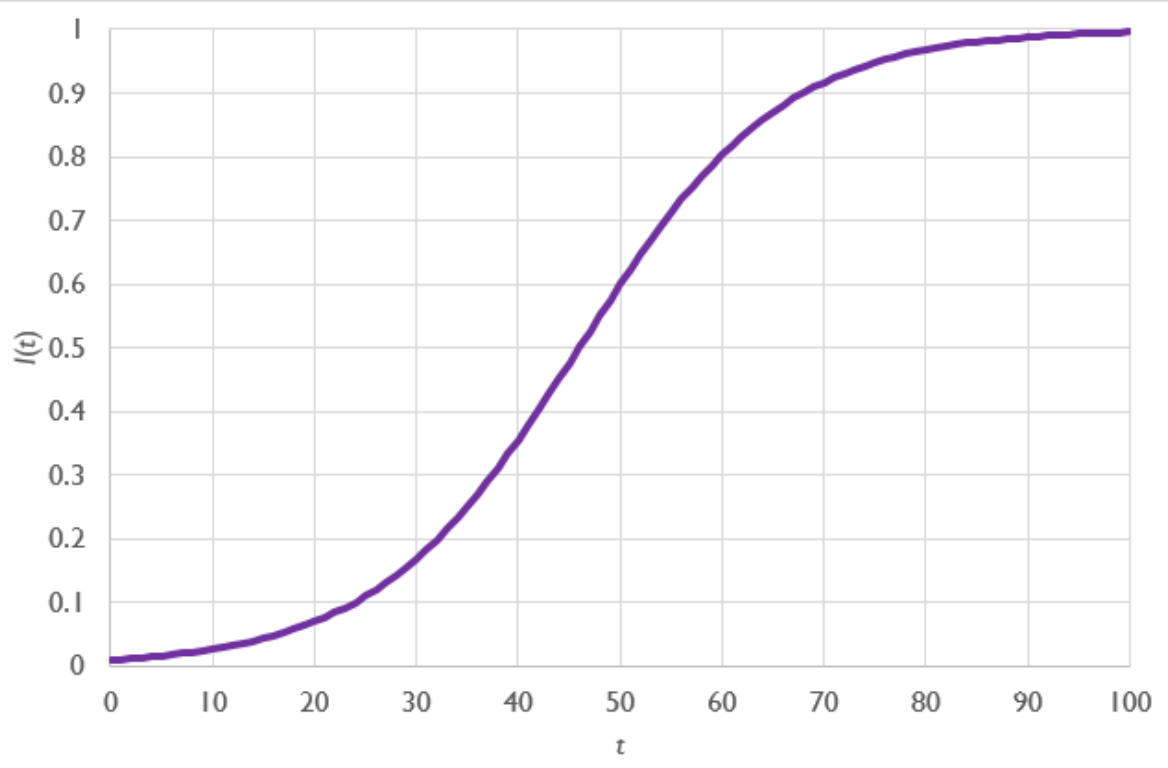

Figure 2: Logistic Growth Curve

We will see in subsequent models that these logistic growth dynamics can be replicated by alternative modelling techniques.

\section{2. (M)S(E)IR Models}

To make the SIS/SIR model more appropriate for specific diseases, Thompson (2016) sets out a comprehensive review of SIR (Susceptible, Infected, Recovered / Removed) models for measles and rubella, with model extensions for other states including $E$ (Exposed but not infected), and $M$ (Maternally Immune) following on from work by Anderson and May $(1979,1992)$. 
Hepatitis B is a virally transmitted disease where a latent time period exists where an infected individual is exposed but not infectious, denoted by $E(t)$ leading to the $S \rightarrow E \rightarrow I \rightarrow R$ model. In the case of measles, babies are not susceptible to measles virus, and are therefore compartmentalized into a $M(\mathrm{t})$ compartment denoting maternal immunity (immunity derived from antibodies transferred from the mother to the baby), and the model becomes $M \rightarrow S \rightarrow I \rightarrow R$.

Further extensions are possible, where additional compartments can be added, for example in the case of tuberculosis, the result of an infection can be that the patient is either recovered or becomes a carrier $C(t)$; the vaccinated population can be added as another compartment $V(t)$.

\subsection{Relationship Between SIR and (M)S(E)IR Models}

It should be noted that the SIR model is a special case of the MSEIR model with the M and E states omitted, and that the SIS model can be easily derived from the SIR model by instead of using a recovered sub-population, these individuals are immediately transferred to the susceptible subpopulation.

Spatial transmission is implicit in SIR models and extensions: these are population level models, and are concerned only with the proportion of the population in each (S, I, R etc.) state, rather than the micro-level interactions that make this transmission possible.

\section{CELLULAR MODELS}

\subsection{Spatial Grids \& Percolation Models}

Grid-based spatial epidemic models have been well studied: Mollison and Kuulasmaa (1985) develop a grid-based SIR model where transmission is made through geographical proximity on the grid. With (2004) studied the effect of ecological landscapes based on percolation with the aim of characterizing landscapes and using these to predict critical thresholds while defining landscape connectivity (With and King 1997). Such 'neutral landscape models' are ecological models, where terrain, soil type, water, and other disturbances, can be mapped into a landscape which accounts for the topology of the landscape. Sikder et al. (2006) consider percolation models, but only in the 
context of a discrete, grid-based percolation system. Atkinson et al. (2008) apply a two-dimensional lattice to consider whether a nuclear-bomb carrying terrorist could travel through a city undetected (modelled by the lattice): a key limitation of their model is the regularity of the modelled system.

\subsection{Cellular Grid Models: The Forest Fire Model}

A simple model of the flow of 'infection' over a wide spatial scale can be introduced by the Forest Fire model (Bak et al. 1990; Chen et al. 1990; Drossel and Schwabl 1992). Several iterations of this model have been developed: we introduce the simplest model of propagation.

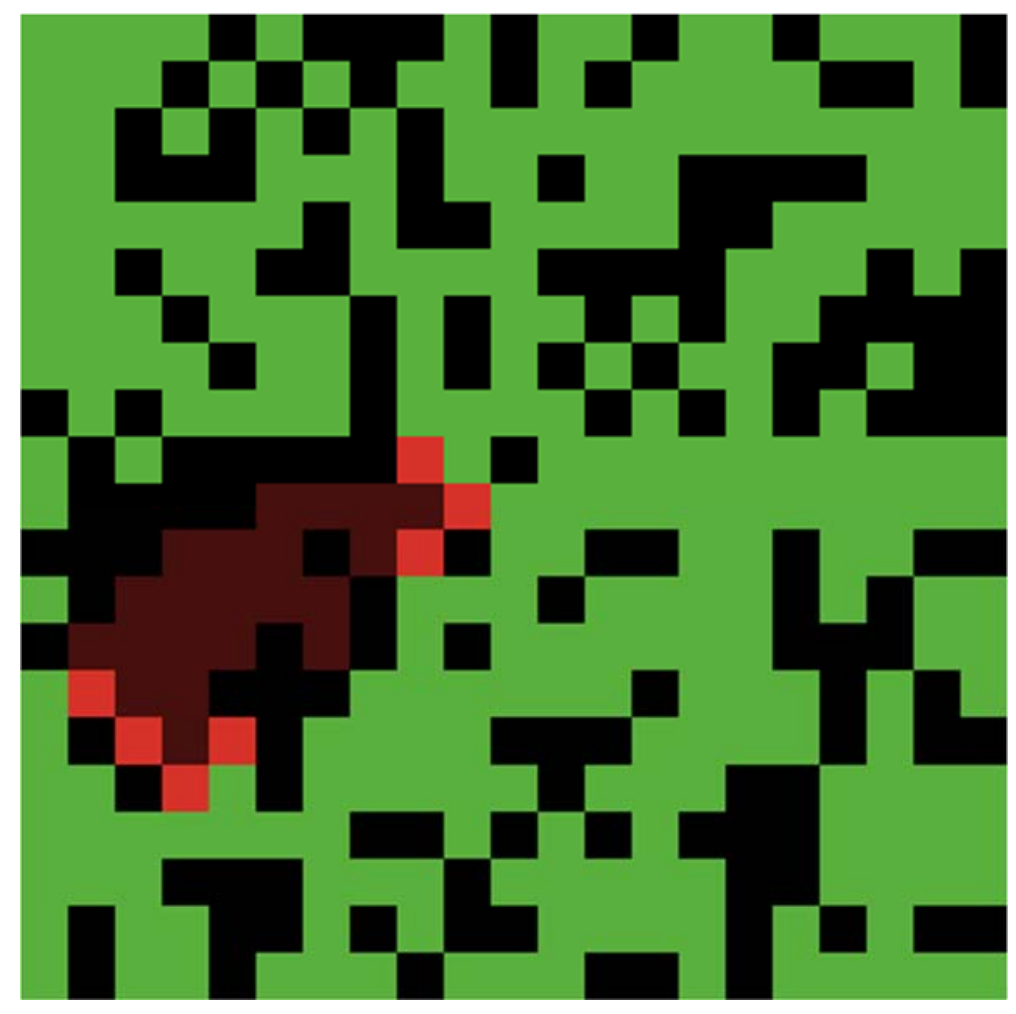

Figure 3: Forest Fire Model showing Progression of a Fire Front and Burnt Trees

The Forest Fire model is situated on a two-dimensional grid (which is used to represent geographical space). Transitions of states between E (Empty), T (Tree), and F (Fire) in these cells takes place with the following probabilities.

$\mathrm{E} \rightarrow \mathrm{T}$ (Tree Growth) - Trees grow with probability $p \Delta t$ 
$\mathrm{T} \rightarrow \mathrm{F}$ (Local Spread) - Fire spreads locally from a tree that is on fire to their tree neighbors with probability $h \Delta t(h \Delta t=1$ for the simple model)

$\mathrm{T} \rightarrow \mathrm{F}$ (Lightning Strikes) - Fire spontaneously ignites in a tree with probability $f \Delta t$

$\mathrm{F} \rightarrow \mathrm{E}$ (Tree Removal) - A burning tree leaves an empty cell with probability $l \Delta t$ ( $l \Delta t=1$ for the simple model)

where $\Delta t$ is a small time interval through which the simulation steps forward in discrete time steps.

Note that the $\mathrm{T}$ (Tree) and F(Fire) states are analogous to modelling individuals in the Susceptible and Infected states in an analytical SIS model as described in §2.1. The motivation for the Forest Fire Model was to demonstrate self-organized criticality, where the size of the cluster of trees that is burnt obeys a power law (see also Bak et al. (1987) for a full explanation in an earlier sandpile model: in the Forest Fire Model, self-organized criticality is exhibited when both $p \rightarrow 0$ and $f / p \rightarrow 0$ ).

\subsubsection{Relationship between Cellular Grid Models and the SIR Model}

Bancal and Pastor-Satorras (2010) show that when $p=f=0$, we recover the SIR model; when $f=0$ and $p \rightarrow \infty$, we recover the SIS model, while $f=0$ means we recover the SIRS model. Note that this is a micro-level simulation of SIR-type models: there is a direct analog between $S$ susceptible (T in the Forest Fire model); $I$ (F in the Forest Fire model $)$; and $R(\mathrm{E}=$ Removed / Empty in the Forest Fire model).

\subsection{From Cellular Grids to Networks}

It could be argued that the Forest Fire model is too simple in that it limits any agent to having a maximum of four (using von Neumann) or eight (using Moore) neighbors (Batty et al. 1997) (or six neighbors in hexagonal cellular grids where the von Neumann and Moore neighborhoods are equivalent). Physical or social systems are however more complicated: individuals may have many more neighbors than this. 
To avoid the limit on neighbors, a network-based approach can be used. Before introducing networks in more depth in $\S 4.2$, we show here that the Forest Fire model can instead be thought of as a network model. To transform the grid representation into a network representation, we construct an adjacency matrix for the grid (which shows which nodes are linked to which other nodes by means of edges) and construct a network representation as shown in Figure 4(c).

Note that Figure 4 (a)-(c) are merely different ways of representing the same underlying neighbors:

Figure 4(a)-(b) represent these neighbors by means of a cellular representation, and Figure 4(c) represents neighbors by means of a network representation. The same numbered cells/nodes are connected in Figure 4(b) and Figure 4(c). Where in a cellular model, (von Neumann or Moore) neighbors are considered connected, the network model explicitly connects neighboring cells by means of (red) edges between (purple) nodes in Figure 4(c).

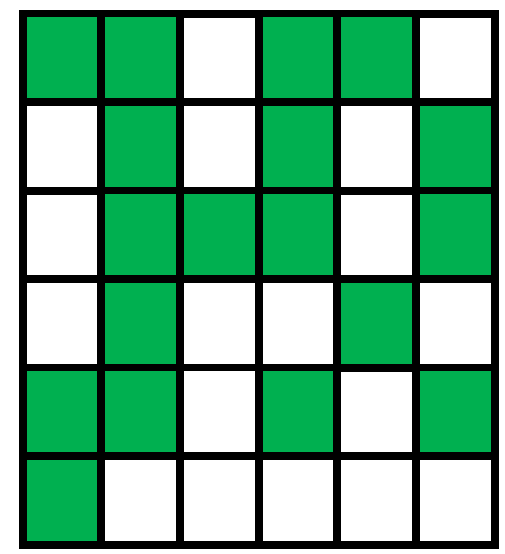

(a)

\begin{tabular}{|c|c|c|c|c|c|}
\hline 1 & 2 & & 3 & 4 & \\
\hline & 5 & & 6 & & 7 \\
\hline & 8 & 9 & 10 & & 11 \\
\hline & 12 & & & 13 & \\
\hline 14 & 15 & & 16 & & 17 \\
\hline 18 & & & & & \\
\hline
\end{tabular}

(b)

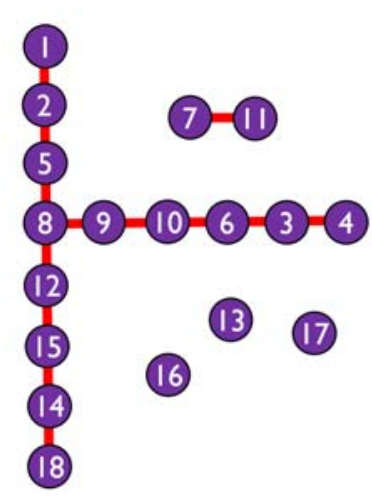

(c)

Figure 4: Forest Fire Grid (a) cells populated with density $\rho=0.5$; (b) cells numbered; (c) network representation

Network representations can be used to model transmission mechanisms, and we show later in $\S 4.3 .1$ that the Forest Fire model is a special case of a Random Geometric Graph network model.

\subsection{Voronoi Cells: From Cellular Grids to Domains}

The reduction of spatial information to an adjacency matrix of network connections may appear to be losing spatial information, but we can transform the network to a geographic representation by use of 
a Voronoi diagram, where domains of each node are generated (i.e. the area $V\left(p_{i}\right)$ for each node $p_{i}$ for which that is the closest node in distance $d$ ), according to the following formula:

$$
V\left(p_{i}\right)=\left\{p \mid d\left(p, p_{i}\right) \leq d\left(p, p_{j}\right), j \neq i, j=1 \ldots n\right\}
$$

This method has been applied to sampling of contaminated soil (Cox 1999) and in exposure to ozone and other pollutants by Fann et al. (2012). The transmission network connections are made from identifying neighboring cells. Figure 5 (after Wilensky 2006) shows a Voronoi diagram for a random distribution of nodes. A precursor of this method was used as long ago as 1854 to identify the source of the London cholera outbreak by plotting the location of water pumps as nodes and identifying the catchment area of each pump as a Voronoi cell (Snow 1855) as show in Figure 5(b). The method of using Voronoi structures for Forest Fire modelling has been used by Shi and Pang (2000) and with extensions in to spatial interactions of shops, residential areas, and cities. This allows an extension of a 2D cellular automata lattice to a more complex geometry.

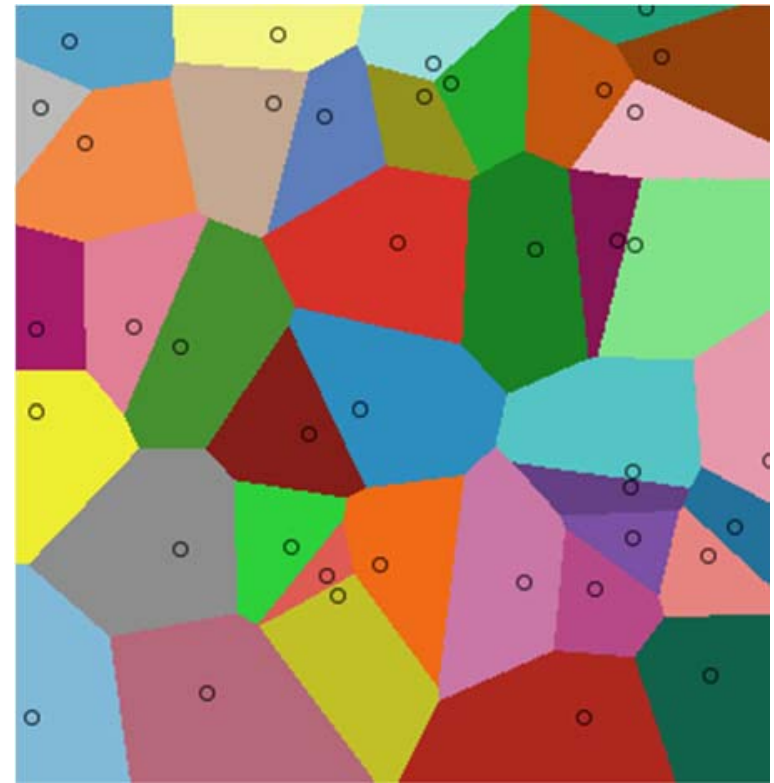

(a)

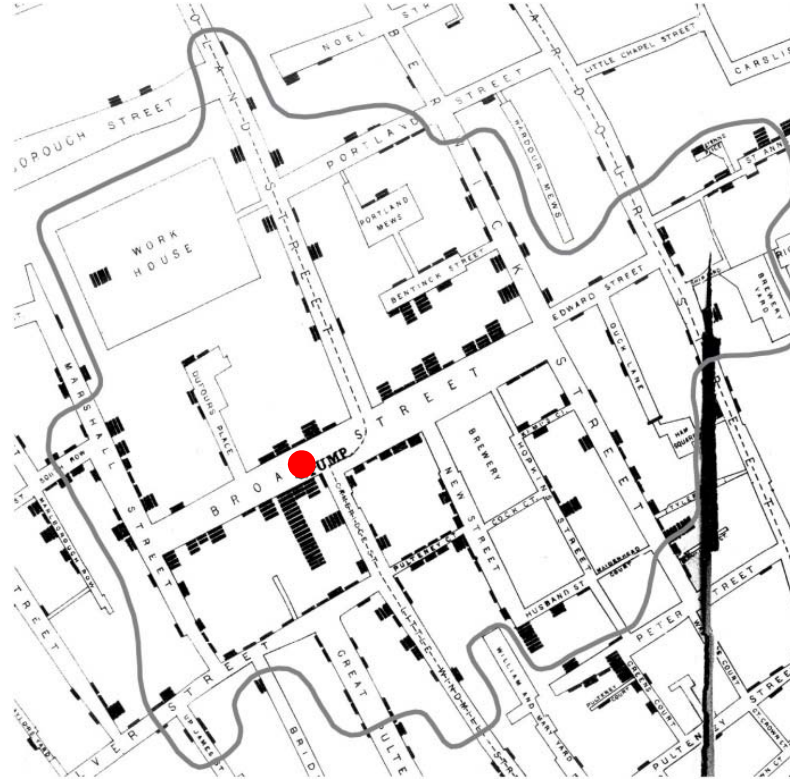

(b)

Figure 5: Voronoi cells (a) generated from a random geometric distribution of nodes; (b) Snow's Cholera mapping with location of pump node highlighted and the Voronoi cell of that pump in grey outline 


\subsection{Delaunay Triangulation: From Domains Back to Networks}

To transform a cellular model into a network model, the Delaunay triangulation can be calculated (Delaunay 1934). This produces a network (shown as red lines in Figure 6). Just as the Voronoi diagram defines the boundaries between nodes into domains, so that every point in the domain polygon is closest to the node residing in that polygon, the Delaunay triangulation connects these nodes so that nodes are linked when there is a boundary between the Voronoi cells of the nodes.

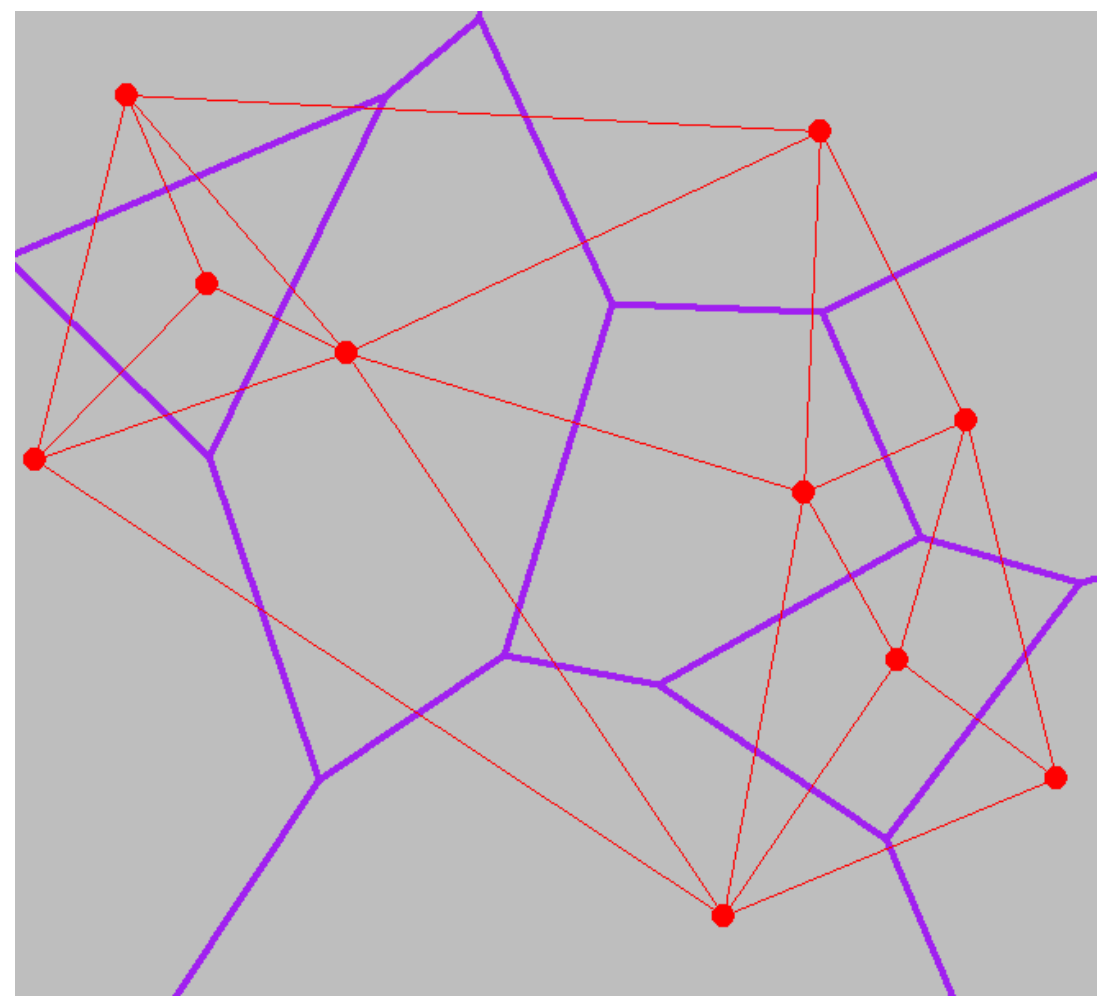

Figure 6: Delaunay Triangulation, generated in $R$ using the deldir package

\section{NETWORK MODELS}

Zio and Sansavini (2011) use network approaches to discuss criticality in network systems. This interesting study uses a geographical network as an example, that of the Italian power grid, but spatial characteristics are lost when transforming a geographic map into a non-geographic network (that is to say the criticality is based purely on the network characteristics, and all spatial information is lost). However, Gastner and Newman's (2006) work shows that the topology of the underlying spatial structure can be 'reverse engineered' from network data, and a more general review of spatial 
networks can be found in Barthélemy (2011). Many empirical and theoretical studies have been performed on the spread of disease over networks such as the airline network (Colizza et al., 2006). We wish to extend this approach from ecological models to a general model for percolation of risk across a general system.

\subsection{From Cellular Grids to Networks: A More General Model of Spatial Risk}

The (grid based) Forest Fire model of $\$ 3.2$ has a limitation that an agent within the system can have a upper bound on the number of neighbors: for example, four in a von Neumann neighborhood. When viewed as a network as in Figure 4(c) this means that the degree (number of connections) of each node is maximum of four. We would like to be able to make a more general model of connections between agents. For this we can turn to more general network theory.

\section{Spatial Graphs}

Networks are collections of nodes, and edges that connect nodes. Much empirical work has been done on the properties of networks, tracing back to Euler's (1736) solution to the Severn Bridges problem - whether it was possible to design a path through the Prussian city of Königsberg (currently the Russian city of Kaliningrad) so that each of its seven bridges would be crossed exactly once. By abstracting away space and converting a spatial problem (that of a city map) into a network problem of only nodes and edges, the problem becomes more tractable.

Figure 7(a) shows a Merian (1652) map of Königsberg with rivers highlighted in blue and the seven bridges in red. There are four spatial regions which are highlighted in yellow in Figure 7(b) - the north, south, and east of the city, and the central island. In order to solve the Seven Bridges problem, the spatial information is abstracted away until we have a graph in Figure 7(c) which can then be analyzed using network techniques.

\subsubsection{Transformation of a Spatial Problem to a Network Model}

In the process of transforming a spatial problem in to a network problem, it is important to note that this remains a planar graph - i.e. one where no edges cross: the spatial relations between the points 
are preserved. This planarity is important when considering spatial problems through network approaches. A graph of a rail or road network will generally be planar, in that when two roads or rails cross, there will (generally) be an intersection. However, a graph of airline transportation network will not - airline routes will cross without an intersection being formed. This does not however preclude treatment of non-planar networks - see Gastner and Newman (2006).

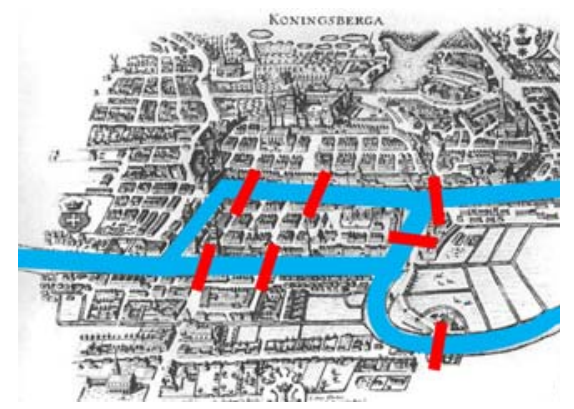

(a)

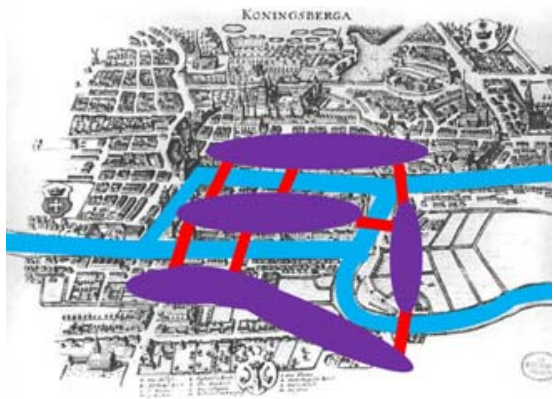

(b)

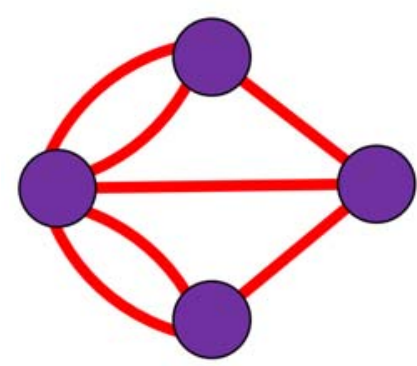

(c)

Figure 7: Euler's Transformation of a Spatial Problem into a Network Problem

\subsection{Network Models}

Many non-planar network generation mechanisms have been proposed, but these may have limited application in spatial risk analysis (assuming we are on a Euclidean space where a map can be generated). Random, Small World, and Scale Free graphs are shown in Figure 8 and described below. In all network models, the connection between nodes may be directed, in which case transmission can take place only in the direction indicated by the arrow, or undirected, in which case transmission may take place in either direction. 


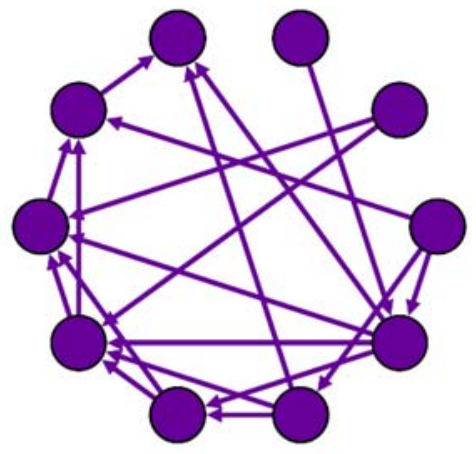

Random Graph

(Directed)

(a)

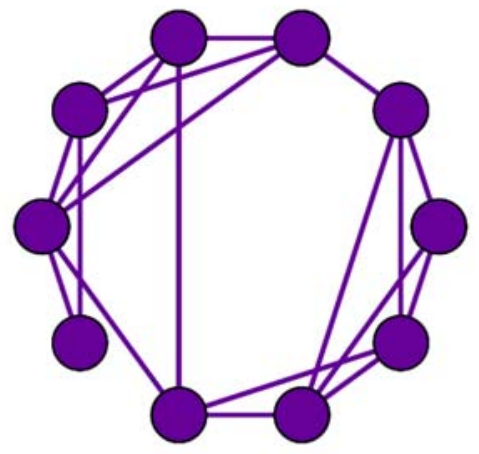

Small World

(Undirected)

(b)

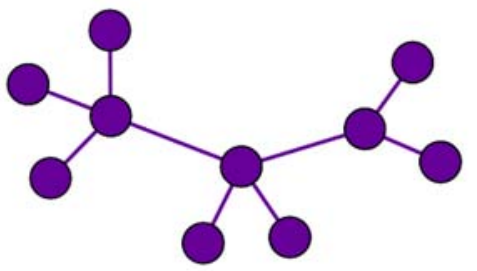

Scale Free (Undirected)

(c)

Figure 8 : Archetypal Network Graphs

\section{Random Graphs}

The Random Graph model was introduced in 1959 by Erdős and Rényi (1959) and independently by Gilbert (1959). An Erdős-Rényi random graph is formed simply by connecting each node with each other node with probability $p$. This however generates non-planar graphs with limited application for spatial analysis (although the Erdős and Rényi random graph generation mechanism has been adapted to a spatial graph by rejecting links that destroy planarity (Barthélemy 2011:39), and as we shall see, the Gilbert random graph becomes a spatial graph under the Random Geometric Graph in §4.3). An issue with the Erdős-Rényi model is that the networks produced do not represent real-world phenomena, in that they do not form local clusters of links, and do not exhibit hubs - this is due to the degree distribution of Erdős-Rényi networks being a Poisson distribution rather than exhibiting a power law.

\section{Small Worlds}

The Small World network model, introduced by Watts and Strogatz (1998) was motivated by the limitations of Erdős-Rényi graphs mentioned above. Milgram (1967) had asked the question as to how many intermediaries were needed to connect two random individuals. In a series of experiments, Milgram identified that the necessary path length was short, which, with earlier challenges by 
Karinthy (1929), helped to popularize the phrase 'six degrees of separation' (Barabási 2002). A Watts-Strogatz Small World network is formed by starting with a ring lattice network of $N$ nodes connected to $K / 2$ neighboring nodes on each side. The regular lattice is then 'rewired' with probability $p$ where $0 \leq p \leq 1$ (the intuition being that when $p \rightarrow 0$, the network becomes fully ordered and when $p \rightarrow 1$, the network tends to a random network). When $N \gg k \gg \ln N \gg 1$, the rewired network exhibits 'small world' properties where there is local clustering and hubs are formed. Watts and Strogatz simulate the spread of a disease on such a network, and note that a small world structure allows a more rapid spread of disease due to the hubs. However, the Small World structure is nonplanar. Prior work had been done by Stoneham (1977), based on a cellular lattice. Jespersen and Blumen (2000) have moderated the Small World network to make links closer in geographical space to have a higher probability of connection, while Kleinberg (2000) and Sen and Chakrabarti (2001) model a two-dimensional grid lattice where connection probability is a function of geographical distance.

\section{Scale Free}

Barabási and Albert (1999) modelled a mechanism for generating networks that exhibit power law ('scale free') the degree $k$ (number of connections from each node) is distributed according to the formula $P(k) \sim k^{-3}$. The mechanism for creating the network is that nodes are added sequentially, with the probability of connecting to a node $i$ depending on the degree of that node, $p_{i}=k_{i} / \sum_{j} k_{j}$ where $k_{i}$ is the degree of node $i$ and $j$ are all existing nodes. As such, preferential attachment occurs, where new nodes are preferentially attracted to nodes with higher degree (number of connections), which gives rise to the phenomenon of super-spreaders (Lloyd-Smith et al. 2005).

Spatial variations of this have been proposed by Kaiser and Hilgetag (2004) where nodes are added randomly in space and the connection of the new node $u$ to existing nodes $v$ were weighted using a probability $P(u, v)=\beta e^{-\alpha d(u, v)}$ where $d(u, v)$ is the Euclidean distance between nodes $u$ and $v$, and $\alpha$ and $\beta$ are constants. This allowed power law distributions of edges to be formed. 
Bancal and Pastor-Satorras (2010) show analytically that under certain parameters, the SIR model of $\S 2.1$ can be recovered from the forest fire model on complex networks.

\subsection{Random Geometric Network / Poisson Point Processes}

The Random Geometric Graph model first introduced by Gilbert (1961) (applying his previous nonspatial (1959) random graph model to a spatial plane) has been used to determine the risk of an adhoc wireless network being infected by a worm virus (Nekovee 2007). Random movement of nodes on the geometric space has been successfully used to model a sexual interaction network where collisions between nodes generate connections (Gonzalez et al. 2006). Further models of connection mechanisms, weakening the deterministic connection when another node is within distance $d$ of the other, and instead modelling 'soft' or 'probabilistic' connections, has been reviewed by Dettmann and Gerogiou (2016). A Random Geometric Graph is shown in Figure 9 below.

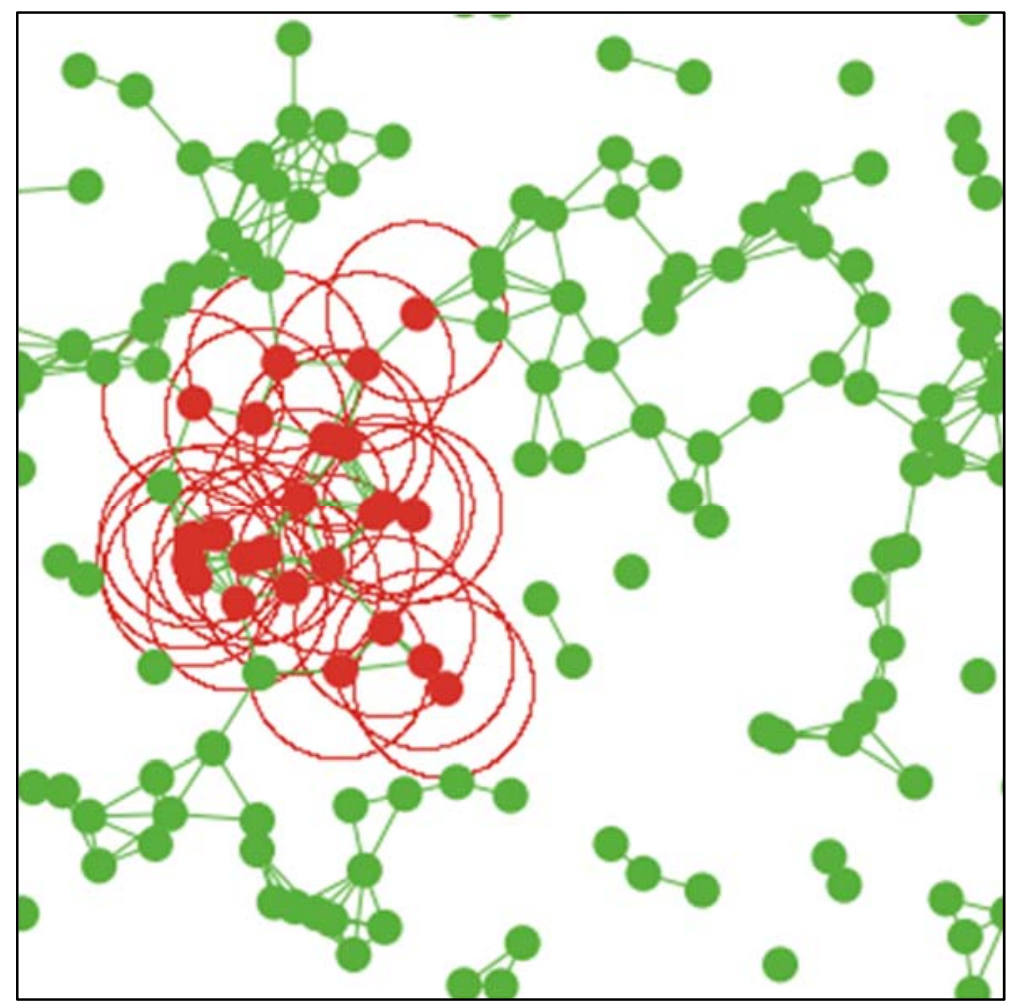

Figure 9: Random Geometric Graph with Uninfected (Green) and Infected (Red) Nodes showing communication distance (red circles) 


\subsubsection{Random Graph Model with Regular Locations Decomposes to a Forest Fire Model}

When we constrain the Random Geometric Graph model to have node coordinates that are regular, we can see from Figure 10 that this decomposes to the Forest Fire cellular model of $\S 3.2$ - we have transformed a cellular model in to a network model. Some work has been done in the physical sciences where these are referred to as lattice networks (Ziff 2009) where percolation (Broadbent and Hammersley 1957) is the process studied, but this representation has not been considered within the spatial transmission field.

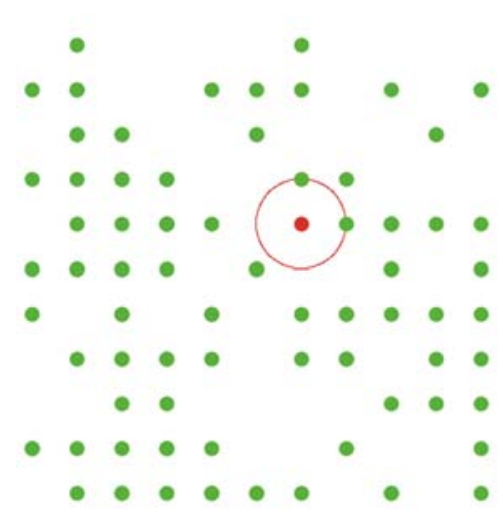

(a)

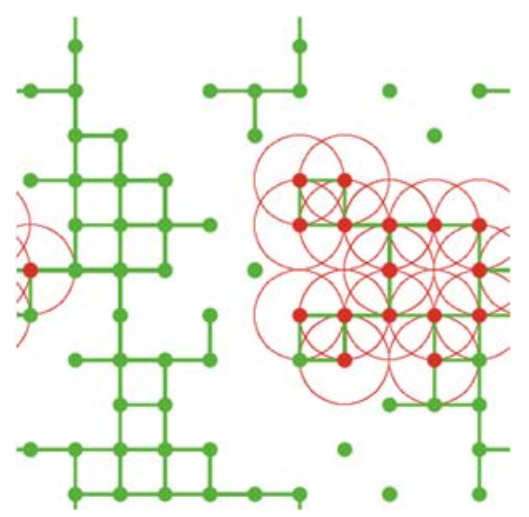

(b)

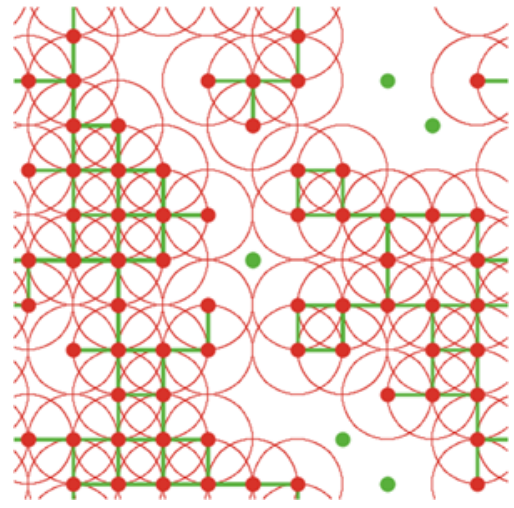

(c)

Figure 10: A Network Representation of the Forest Fire Model by way of a Regular Geometric Graph Model

We need to define a risk metric for network models. Fortunately, a great deal of work has been done in the physical sciences on percolation - when long range connections are made through a chain of local interactions - meaning that local links can be transformed into global links across the entire system. The application to risk is summarized by Robert A. Frosch, the fifth Administrator (head) of NASA, in his testimony to the National Academy of Engineering Committee on Accident Precursors wrote about system failure and identified self-organized criticality and percolation as a model and mechanism through which systems fail (Frosch 2004) - the idea being that small errors occur with large probability, and large errors occur with low probability (and follow a power law distribution) what has to be prevented is a series of small errors cascading into a catastrophic error. 


\subsubsection{Random Geometric Graph Exhibiting SIR Trajectories}

We study the percolation threshold of the Random Geometric Graph by determining for which connection distance $d$ does the system connect fully - when all nodes can be reached from all other nodes. This is termed a giant cluster. We run a series of repetitions of the model, starting a transmission at a random node, and then spreading to neighbors within the connection distance $d$. We repeat this connection mechanism until no more connections can be made, and determine whether the network has formed a giant cluster where all nodes are connected. We plot the probability of a giant cluster forming as a function of the number of nodes and the connection distance $d$ in Figure 11 .

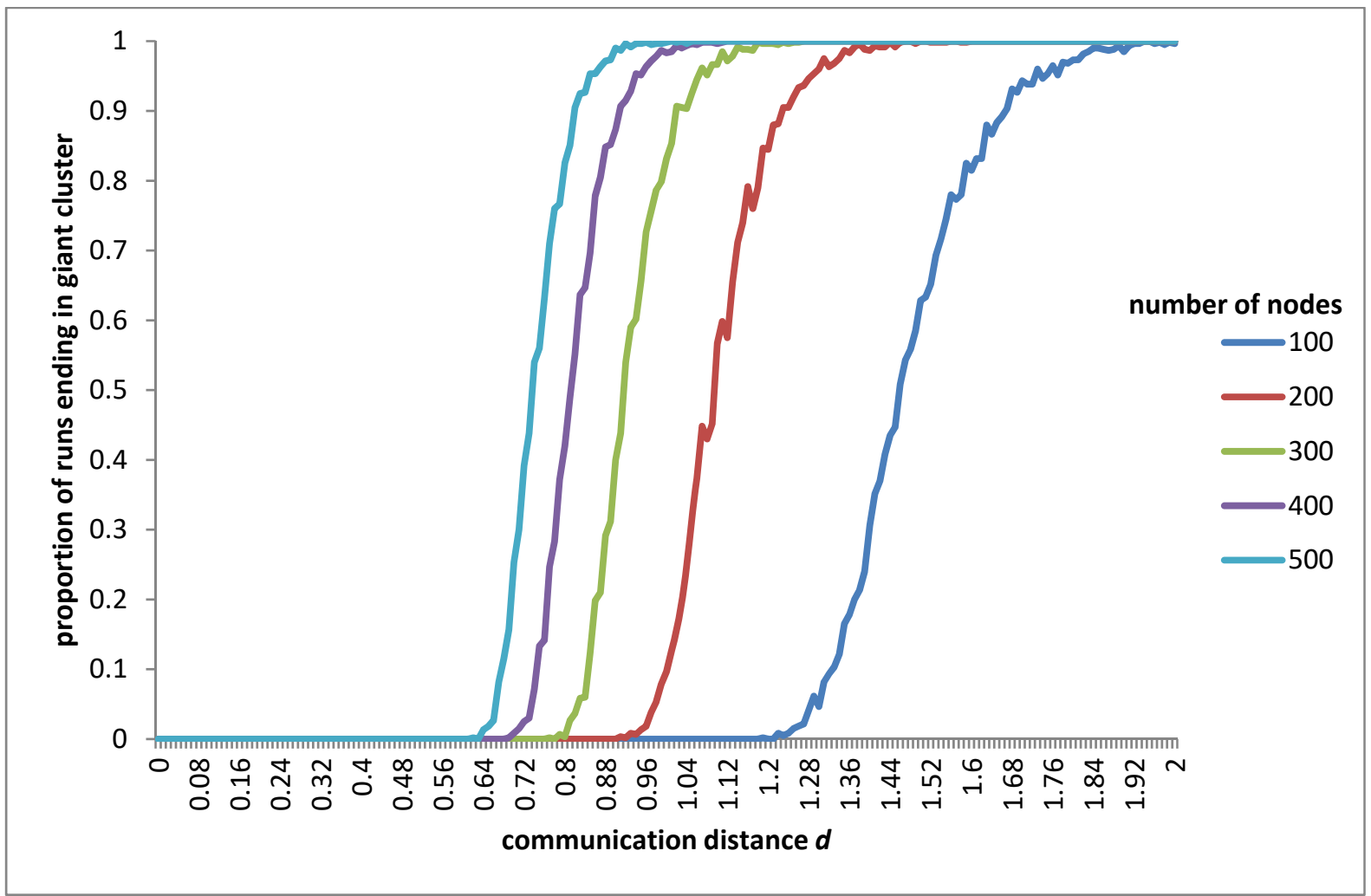

Figure 11: Percolation from Simulation showing critical points and phase transition

We further study the percentage of nodes that are connected (rather than measuring whether a giant cluster has formed or not) as a function of time. Results from these experiments are shown in Figure 12. We see that these exhibit SIR-like infection logistic curve trajectories (as shown analytically in Figure 2), with the asymptotic limit dependent on the density of connections. 


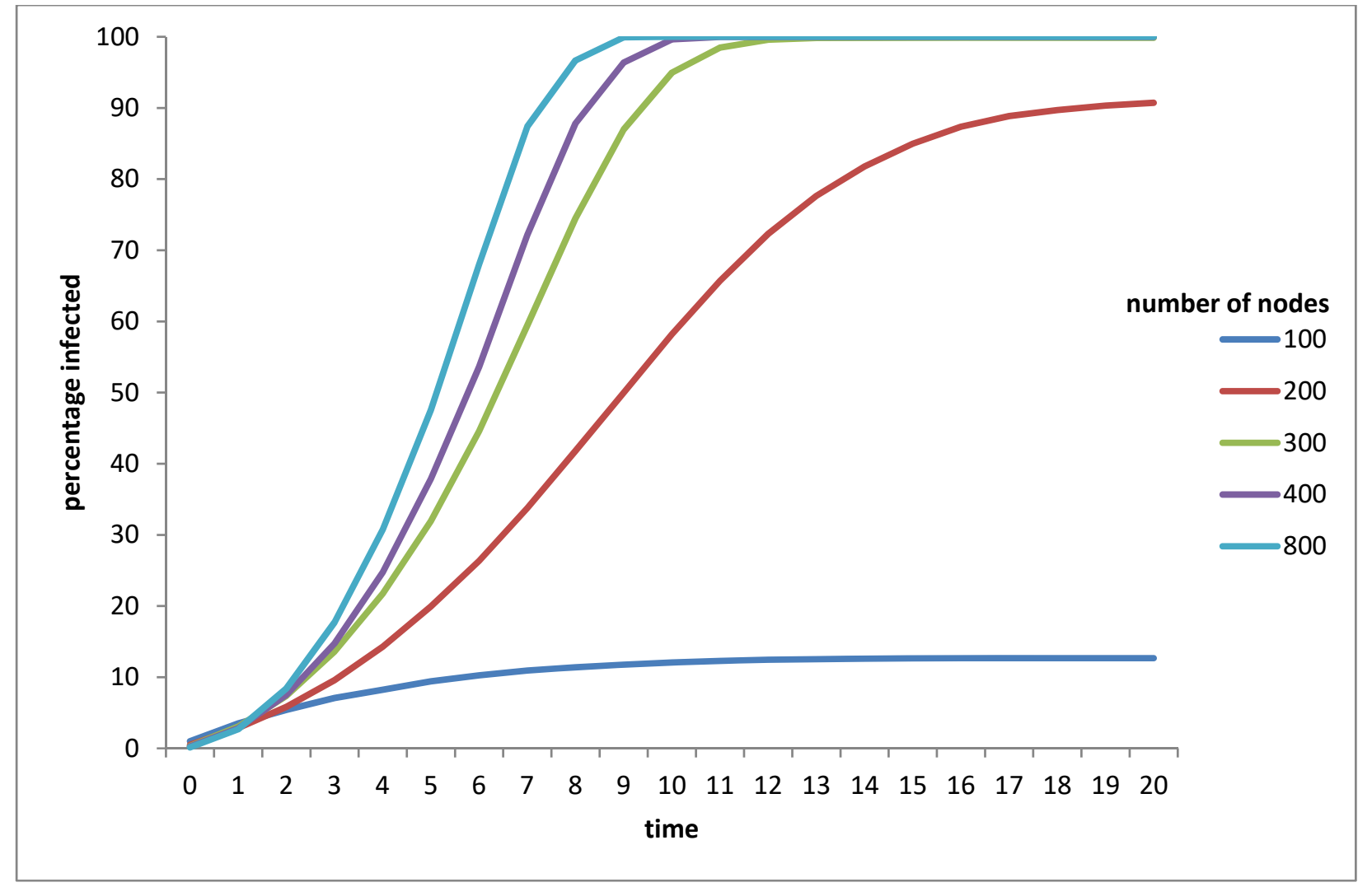

Figure 12: Percolation over time showing SIR-like behaviour

\section{AGENT MODELS}

\subsection{From Networks to Agent-Based Models}

Instead of abstracting away the spatial elements in a network model in $\S 4$, we can instead have the spatial coordinates of these nodes as an integral part of the model. By treating nodes as agents, we can generate an agent-based model, where we model individual, possibly heterogeneous, agents, and give them characteristics that allow more complex and sophisticated interactions, where more nuanced transmission mechanisms can be investigated.

Agent based models have been used in risk studies for bank systems (Robertson 2003), economic agents (Holland and Miller 1991), and road traffic (Erol et al. 2000) amongst others. A wide range of toolkits can be used to develop agent-based models - for a review of toolkits for agent-based modelling, see Robertson (2005). 
Agent-based models, instead of modelling the system at a system level, model individuals or groups of individuals at a micro level. These 'agents' are heterogeneous, but can be modelled as homogeneous agents by restricting the variability in the agent parameters. Agent parameters can include age, energy levels, location in space, rules of behavior etc.

Agent-based models can be used to model SIR-like systems of $\$ 2.1$ on top of a geographical space (using GIS data - §5.2 - or a stylized map). They can also be used more generally to model the transmission of 'information' - be it virus spread leading to population-level infection; discontent leading to civil unrest; fire leading to forest fires; or terrorists moving through a city to set off a nuclear device. Policy interventions can be modelled using system-level parameters, for example the number and locations of respectively, culling regions for disease; of pro- or anti-Government propagandists; of fire breaks; police officers deployed. Figure 13 shows an agent-based model where transmission takes place by local interaction between agents.

In the agent-based model in Figure 13, agents are in one of two states: susceptible (green) or infected (red). Agents move randomly around the space, and if they are then in the proximity of an infected agent, they transition between susceptible and infected states. The agent-based model has similarities and differences to the random geometric graph model in $\S 4.3$ : transmission takes place through a transmission radius, where the distance between agents is measured, and if a susceptible agent is within radius $r$ of an infected agent, they will become infected (either deterministically or with a probability that depends on $r$ ). The transmission in the agent-based model in Figure 13 is by movement of the agents themselves: the agents themselves can move around space and in doing so, infect susceptible agents. The agent-based model is also more versatile: each agent can be heterogeneous in that they can have different susceptibility to infection, different durations in which they remain infected (a recovered state can also be included when agents transition from infected to recovered). Entrants and exits of agents to/from the model can also be modelled. 


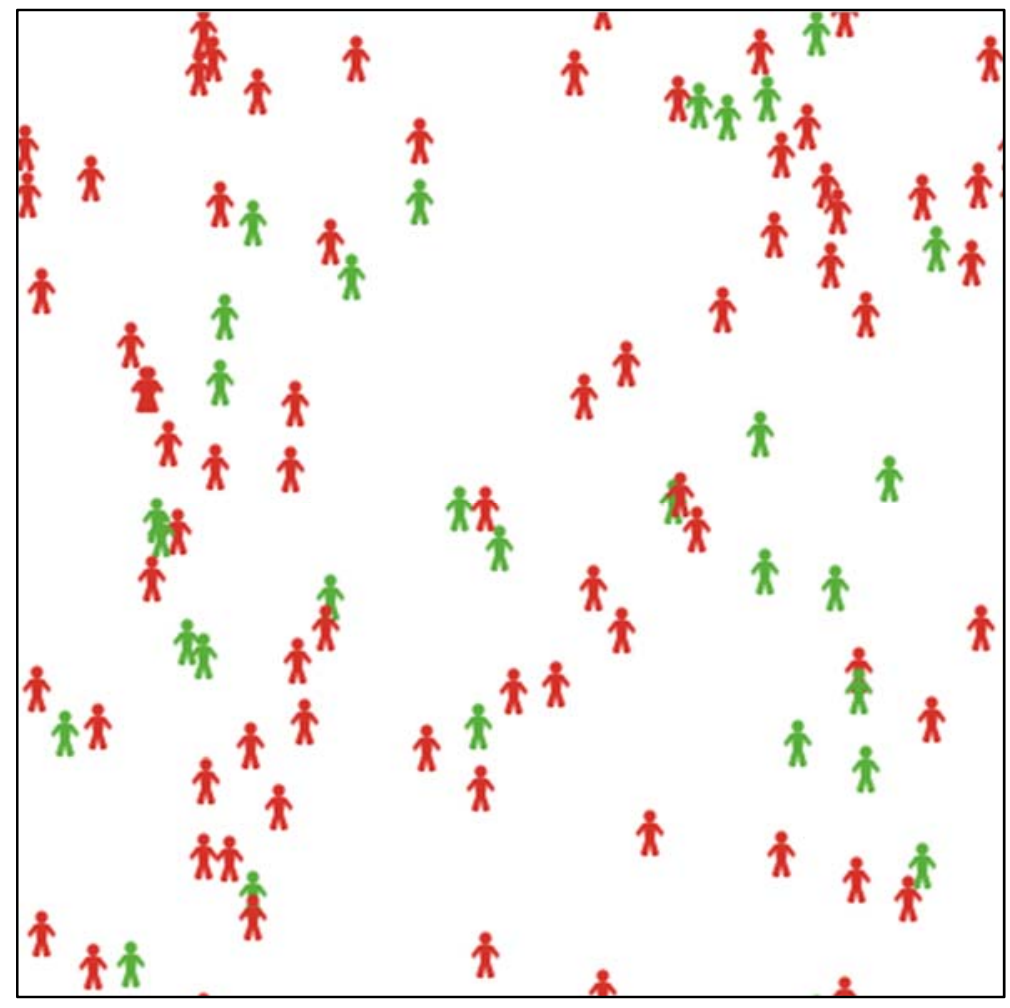

Figure 13: Agent-Based Model showing agents with different states

Fundamentally, the fact that agents are (at least in most models) physically located in space, makes them an ideal candidate for modelling risk in spatial systems. As with the models above, changes to parameters such as agent density can have a critical, non-linear effect on the system as a whole, and such effects may not be able to be modelled using alternative approaches. Indeed, the landscape can be deformed by the presence of agents (e.g. Robertson and Caldart 2009).

Agent-based models can build on and do not necessarily replace the models described in earlier sections of this paper. Indeed, reducing the complexity of agent behavior (e.g. restricting the location of agents in space and restricting the possible connections between agents) means that earlier systems can be modelled using agent-based models by not taking advantage of the benefits of modeling agent heterogeneity. 


\subsubsection{From Agent-Based Models to Network Models}

Agent-Based Models can be combined with network models, so that the transmission network is generated from the locations of the agents (which can move over time meaning a dynamic network is created).

Figure 14 shows an endogenously created social network where social network relations are created by reference to height on a landscape (represented by the depth of purple color). In this model, agents compare their height to their nearest neighbor, and if their height is within a height threshold of their neighbor, they will stop moving; if the threshold is not reached, the agents will move randomly across the space. The arrows in Figure 14 show the direction of comparison at equilibrium (when all agents have stopped moving). In this way, a social network of connections is generated endogenously, which has the side effect of splitting the space into unconnected social network regions similar to the domains of Voronoi cells in $§ 3.4$.

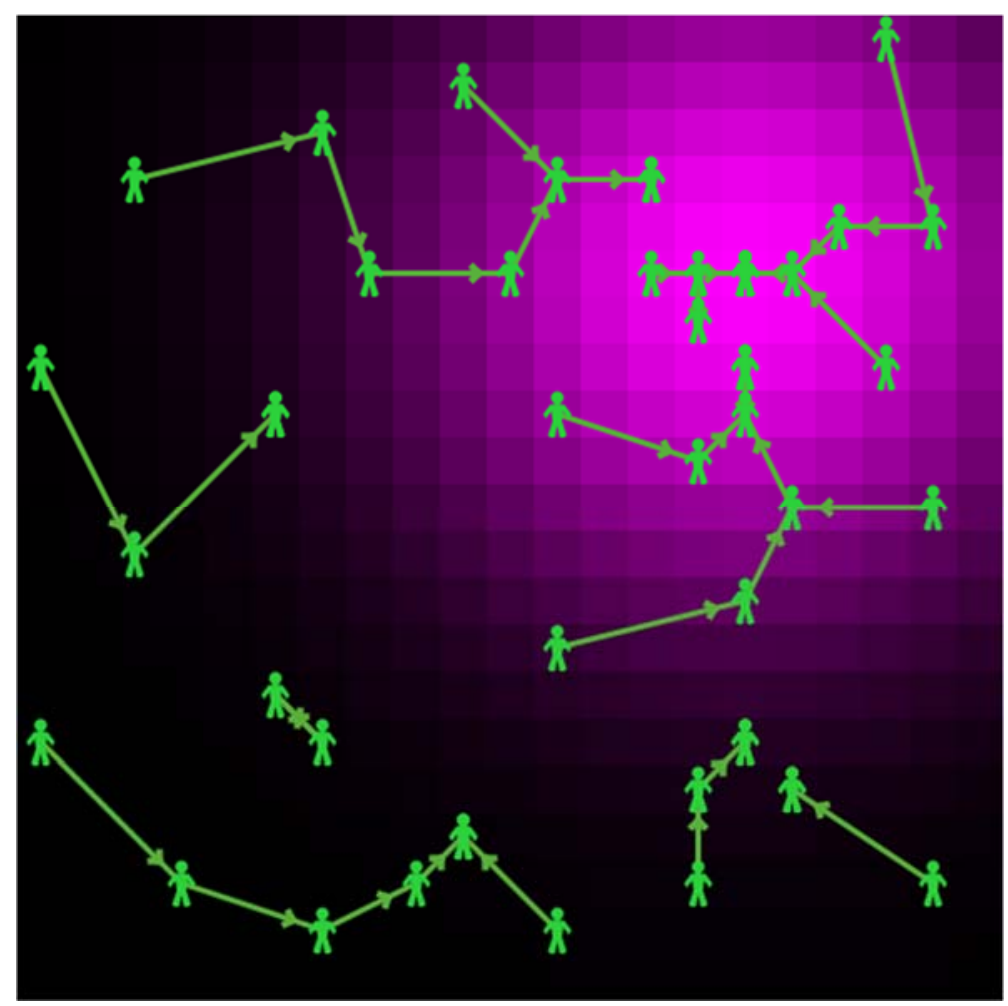

Figure 14: Social Network Created Endogenously in an Agent Based Model 


\subsection{From Agent-Based Models to Agent-Based GIS Models}

Figure 15 (after Wilensky 2017) shows a Geographic Information System ('GIS') where landscape and rivers are shown as a multi-layered landscape. Agents are able to traverse the landscape, taking into account attributes of the geographic space such as land type, hydrological features, topology, etc. For a review of a GIS adaptation of Snow’s cholera map discussed in $§ 3.4$, see Koch (2004). Uses of GIS and Agent-Based Models include Crooks and Wise's (2013) analysis of aid distribution following natural disasters.

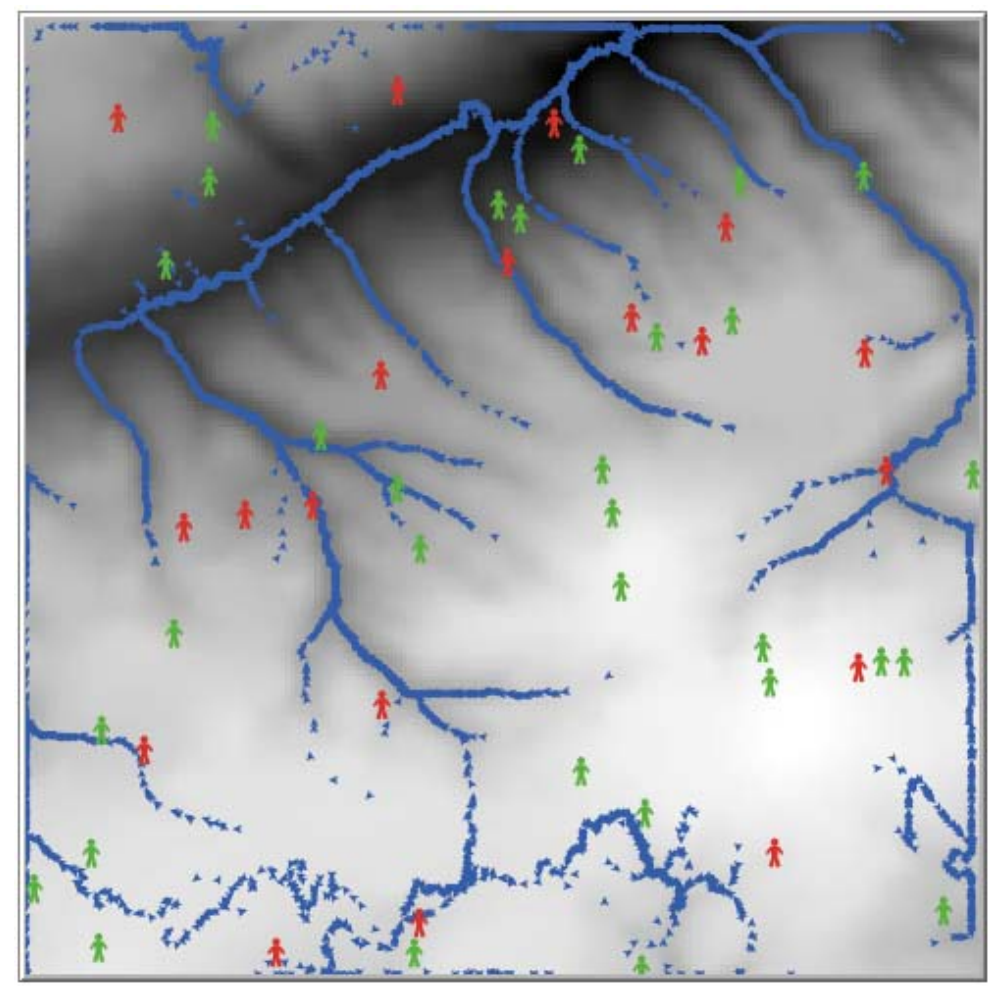

Figure 15: Agent-Based Model on a GIS Landscape

\subsubsection{GIS Models and Agent-Based Models}

There is a debate as to whether Agent-Based Models on GIS landscapes should be considered separate from agent-based models. Heppenstall et al. (2011) provide a field review of agent-based models of geographical systems. Torrens and Benenson (2005) consider the explicit integration of GIS and ABM in 'geographic automata systems', which merge agent-based modelling and GIS within a 
specialized modelling environment. However, several authors take the view that GIS data should be incorporated into ABM software. Brown et al. (2005) discuss this debate further, considering how GIS and ABM should be integrated, concentrating on a 'middleware' approach which links together GIS and ABM modelling platforms, particularly systems such as RePast Symphony, which integrates open source GIS, specifically GeoTools (North et al. 2013). More recent work has used shapefiles from GIS data to generate polygons as the topology upon which an agent-based model such as the Schelling model (§5.3) can be run (Zhou 2015).

\subsection{From Agent-Based Models back to Cellular Models}

The transmission within cellular grid models uses simple rules (for example, a cell will become infected if next to an already infected cell), but these rules do not allow for more complex transmission or the movement of hosts. More recent grid models, inspired by complexity science, have used models where hosts can move. In the Schelling model of spatial segregation (Schelling 1971), the hosts are modelled as individual agents, each with their own identity. By varying the agents' proclivity for having neighbors of the same color, segregation of the agents is found to occur, meaning that the hosts move around the system. In this way, dynamics on the cellular grid model are extended to include movement of hosts on the cellular grid.

One major advantage of agent-based models is that they are dynamic: agents can adapt to a changing environment. One of the concepts behind complex systems modelling is the concept of emergence (Goldstein 1999) - the properties of a macro system generated by the micro-level interactions between the actors in the system. Schelling (1978) was one of the first to identify this emergence in his book Micromotives and Macrobehaviors based on the spontaneous racial segregation observed in Chicago. With only one parameter - the percentage of neighbors of the same color - segregation (a macro scale property) takes place without the input from a social planner: the macro level property is generated from micro level interactions. 


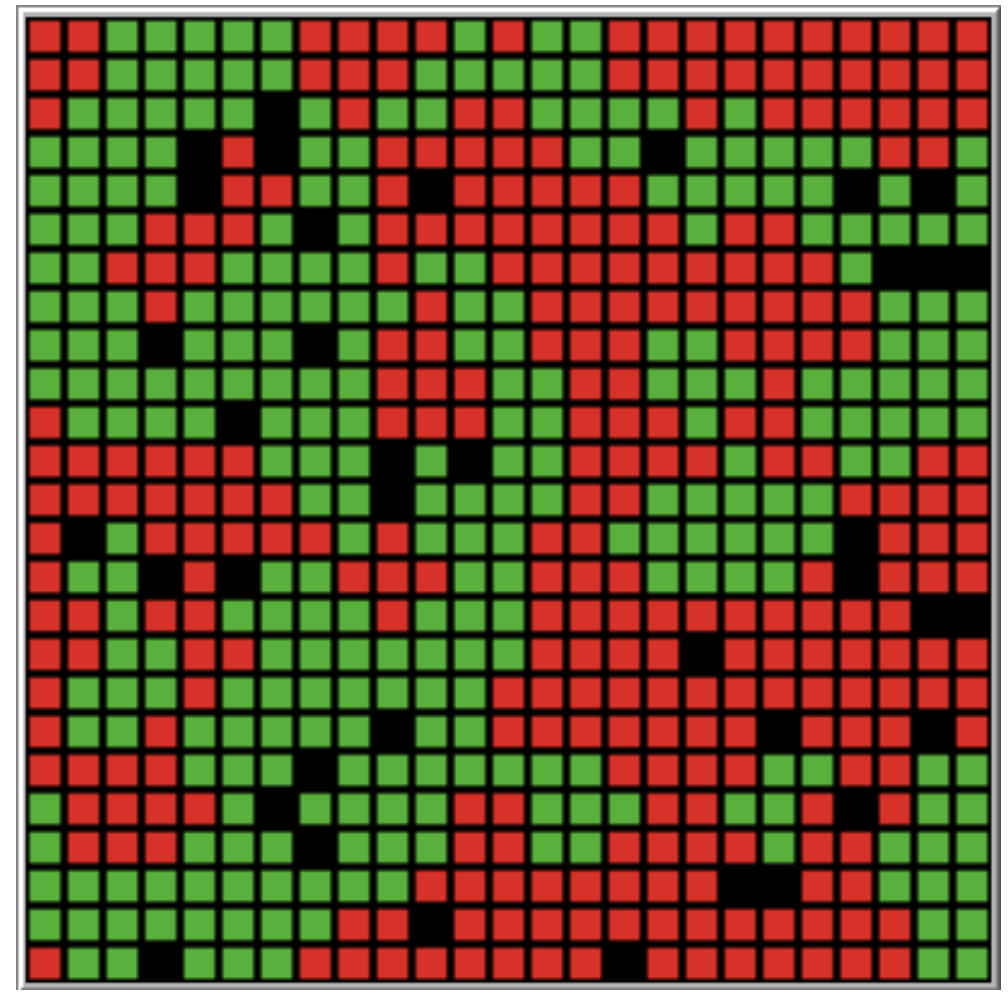

Figure 16: Schelling Segregation Model

\section{A TAXONOMY OF SPATIAL TRANSMISSION MODELS}

The decision as to which modelling framework (population, cellular, network, or agent) to choose is a difficult one, and we build on previous work (Brennan et al. 2006, Riley 2007) to compare the pros and cons of the disparate modelling techniques introduced in this paper. This allows a modeler faced with a problem of spatial modelling to determine at a glance whether a choice of modelling technique is appropriate for the particular problem that is being studied. This can be used in conjunction with the links between modelling frameworks introduced in $\S 7$ to determine whether other methods may be more appropriate.

In Table 1, we set out model characteristics and compare modelling techniques introduced in this paper. In the table, the transmission route indicates how the transmission takes place, whether modelled as a neighboring spatial interaction or from node to node in a network; transmission interface describes the boundary between hosts; host mobility specifies whether hosts are mobile or are assumed to be static; spatial locations of hosts shows whether hosts are constrained to particular 
positions such as on a grid, domain, within a network, or on continuous or GIS space. Note that population (e.g. SIR) models and agent-based models are different: population models do not explicitly model spatial locations (except when sub-divided into spatial compartments), and agentbased models are versatile in that they may model transmission through the movement of hosts, transmission vectors, or neither.

\section{Transmission Route \& Transmission Interface}

The transmission route and transmission interface determine how transmission is considered in the model. In the case of a population that becomes infected, it may not be necessary to explicitly model the nature of that transmission: the information that is of interest is the proportion of the population that is infected rather than the spatial distribution. In this case, population models can be used. Where however transmission takes place from neighbor to neighbor, the unit of analysis must be decided. We could decide to model each individual agent, but modelling domains may be sufficient, for example transmission from town to town or field to field. In this case, cellular models can be used. Where local transmission occurs, this can either be modelled via network models (Eubank et al. 2004) or via modelling the individual host movements. The decision as to which modelling framework to use is as much a decision as to the necessary level of abstraction and the unit of analysis: population, geographical region, connection, or individual.

\section{Host Mobility \& Spatial Locations of Hosts}

Most transmission takes place where hosts are mobile, i.e. can move in space. However, whether this mobility needs to be considered as part of the modelling process, or whether it can be abstracted away, can determine the most suitable type of model to be used: the movement of individuals can be an important factor in the transmission of a pathogen (Stoddard et al. 2009). If hosts move slowly, or the transmission speed is much faster than what we will refer to as the 'drift speed' (i.e. the averaged net movement speed) of the hosts, the movement of individual hosts need not necessarily be considered, although the mixing of individuals within a domain may be important in determining the persistence within that domain (Cross et al. 2005). Similarly, the location of hosts may be (a) not 
considered, (b) within a grid or network structure, (c) within a continuous space, or (d) taken from geographic information system (GIS) data. Hosts may also be modelled as being constrained within a domain, and host mobility may be considered only at the domain boundary, so that movement from one domain to the next is considered, not intra-domain movement.

\subsection{Multimethod Models}

Modelling techniques have been described in this paper as being independent but linked through the connections shown in Figure 17. Models can however be developed that use more than one method within the same model. Shanthikumar and Sargent (1983) provide a classification of 'hybrid' models where the interaction is between a simulation model and an analytical model, although this can be extended to models where there is interaction between two different modelling techniques. The Shanthikumar and Sargent classification can be generalized as follows: multimethod models alternate between two independent models; multimethod models run in parallel with interactions through their solution procedures; multimethod models use a modelling technique subordinate to another; and multimethod models require inputs from the solution procedure of another method. A variety of these combinations within multimethod models that link Agent and Population models include Tekippe and Krejci (2016) for psychology; and Helel et al. (2007) for manufacturing systems. The link between Agent and Network models is made by models such as Carley's BioWar simulation (Carley et al. 2006), while the link between Population and Network models is made by authors such as Sloot et al.'s (2008) study of HIV transmission. The link between SIR Population models and Cellular models has been made by White et al. (2007), while SIS Population models have been developed in a Cellular model (Boccara and Cheong 1993). The link between Cellular and Agent models, the final combination, is less strong, as cellular models can be considered a more restrictive subset of Agent models, so that a combination of these could be considered to be a Cellular model subsumed into an Agent model: for a review, see Clarke (2013).

More than one technique can be used to model the same system, and these models can be aligned. We leave this more formal 'alignment of computational models' or 'docking' (Axtell et al. 1996) of the 
spatial transmission models introduced in this paper for future research. While comparisons of two modeling techniques to model the same system have been performed by authors such as Rahmandad and Sterman (2008) and Morecroft and Robinson (2005), the alignment of more than two of the modelling techniques described in this paper opens up a rich area for future research. 


\begin{tabular}{|c|c|c|c|c|c|c|c|c|}
\hline Type & POPULATION & \multicolumn{3}{|c|}{ CELLULAR } & \multicolumn{2}{|c|}{ NETWORK } & \multicolumn{2}{|c|}{ AGENT } \\
\hline Model & $\begin{array}{l}\text { SI/SIS/SI/ } \\
\mathrm{M}(\mathrm{S}) \mathrm{E}(\mathrm{I}) \mathrm{R}\end{array}$ & Cellular Grids & Voronoi Cells & Network CA & Network models & $\begin{array}{c}\text { Geometric } \\
\text { Network / PPP }\end{array}$ & Spatial ABM & ABM on GIS \\
\hline $\begin{array}{l}\text { Section } \\
\text { Discussed }\end{array}$ & $\S 2.1$ / \$2.2 & $\$ 3.2$ & $\S 3.4$ & $\$ 4.3 .1$ & $\$ 4.2$ & $\S 4.3$ & $\S 5.1$ & $\S 5.2$ \\
\hline Representation & $\frac{d I}{d t}=\cdots$ & & & !I! & & $88^{\circ}$ & 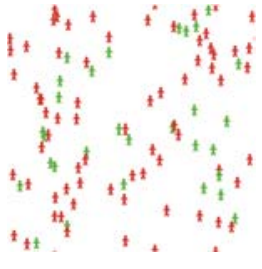 & \\
\hline $\begin{array}{c}\text { Transmission } \\
\text { Route }\end{array}$ & Population Level & $\begin{array}{c}\text { Spatial } \\
\text { Neighbors }\end{array}$ & $\begin{array}{c}\text { Spatial } \\
\text { Neighbors }\end{array}$ & $\begin{array}{l}\text { Network } \\
\text { Neighbors }\end{array}$ & $\begin{array}{l}\text { Network } \\
\text { Neighbors }\end{array}$ & $\begin{array}{c}\text { Spatial } \\
\text { Neighbors }\end{array}$ & $\begin{array}{l}\text { Either Spatial } \\
\text { Neighbors with }\end{array}$ & $\begin{array}{l}\text { Either Spatial } \\
\text { Neighbors with }\end{array}$ \\
\hline $\begin{array}{l}\text { Transmission } \\
\text { Interface }\end{array}$ & $\begin{array}{l}\text { Implicit (Not } \\
\text { Modelled } \\
\text { Explicitly) }\end{array}$ & $\begin{array}{l}\text { Neighbors on } \\
\text { Domain } \\
\text { Boundary }\end{array}$ & $\begin{array}{l}\text { Neighbors on } \\
\text { Domain } \\
\text { Boundary }\end{array}$ & $\begin{array}{c}\text { Neighbors } \\
\text { connected by } \\
\text { network links } \\
\text { (edges) }\end{array}$ & $\begin{array}{c}\text { Neighbors } \\
\text { connected by } \\
\text { network links } \\
\text { (edges) }\end{array}$ & $\begin{array}{l}\text { Neighbors within } \\
\text { distance } d\end{array}$ & $\begin{array}{l}\text { distance } d \text { or } \\
\text { explicit modeling } \\
\text { of transmission } \\
\text { vectors }\end{array}$ & $\begin{array}{l}\text { distance } d \text { or } \\
\text { explicit modeling } \\
\text { of transmission } \\
\text { vectors }\end{array}$ \\
\hline Host Mobility & $\begin{array}{l}\text { Implicit (Not } \\
\text { Modelled } \\
\text { Explicitly) } \\
\end{array}$ & $\begin{array}{l}\text { Fixed within } \\
\text { Domain }\end{array}$ & $\begin{array}{l}\text { Fixed within } \\
\text { Domain }\end{array}$ & Not Mobile & Not Mobile & Not Mobile & Mobile & Mobile \\
\hline $\begin{array}{c}\text { Spatial } \\
\text { Locations of } \\
\text { Hosts }\end{array}$ & $\begin{array}{l}\text { Implicit (Not } \\
\text { Modelled } \\
\text { Explicitly) }\end{array}$ & Grid & $\begin{array}{l}\text { Irregular } \\
\text { Domains }\end{array}$ & Grid & Network & Continuous & Continuous & GIS \\
\hline $\begin{array}{l}\text { Example } \\
\text { Uses }\end{array}$ & $\begin{array}{c}\text { Thompson } \\
(2016)\end{array}$ & $\begin{array}{c}\text { Atkinson et al. } \\
\text { (2008) }\end{array}$ & $\begin{array}{l}\text { Shi and Pang } \\
\text { (2000) }\end{array}$ & $\begin{array}{c}\text { Ziff } \\
(2009)\end{array}$ & $\begin{array}{c}\text { Eubank et al. } \\
\text { (2004) }\end{array}$ & $\begin{array}{l}\text { Nekovee } \\
\text { (2007) }\end{array}$ & $\begin{array}{l}\text { Robertson and } \\
\text { Caldart (2009) }\end{array}$ & $\begin{array}{c}\text { Crooks and Wise } \\
\text { (2013) }\end{array}$ \\
\hline
\end{tabular}

Table 1: Overview of Types of Spatial Models 


\section{A FRAMEWORK FOR SPATIAL TRANSMISSION MODELLING APPROACHES}

There is a tension between simple, tractable models for risk analysis, and complex models with many parameters. The use of simple models to produce interesting results has been advocated by Zagmutt et al. (2016:951). However, in the context of risk within a spatial setting, mechanisms for transmission may be non-trivial. Seminal risk analysis papers have identified the 'social amplification' (Kasperson et al. 1988) of risk where there is a transition from a well-ordered system into a system that is out of control. These transitions or 'tipping points' are not easily captured by traditional models for the analysis of risk. In the physical sciences, one measure of risk is that of percolation transitions - when a system becomes 'critical'. Such critical systems are seen in stock market crashes where local action cascades to a failure of the entire system, or propagation of small errors such as in space exploration disasters. A range of different modeling techniques should therefore be available to practitioners so these phenomena can be captured where they exist.

To this end, we introduce a framework for assisting in deciding which modelling technique can be used for a particular spatial transmission problem. Figure 17 sets out this framework. A series of questions (in blue diamonds) leads to an appropriate modelling technique, shown by red rectangles. An important point to note is that these modelling techniques (labelled as red rectangles) can, in certain circumstances, revert to each other, and therefore provide a pathway to alternative modelling techniques that can be considered by the modeler. These links are shown as dotted purple links between the modelling techniques. For each modelling technique and link between these techniques, a reference is made to the section number below, where the technique is described in more detail.

The choice of technique is however not prescriptive, and the dotted purple lines show relationships between the techniques. It is therefore possible that an alternative modelling technique can be chosen if appropriate. For example, to model the logistic curve (see Figure 2), any of the techniques shown may be chosen depending on the required level of model granularity. 


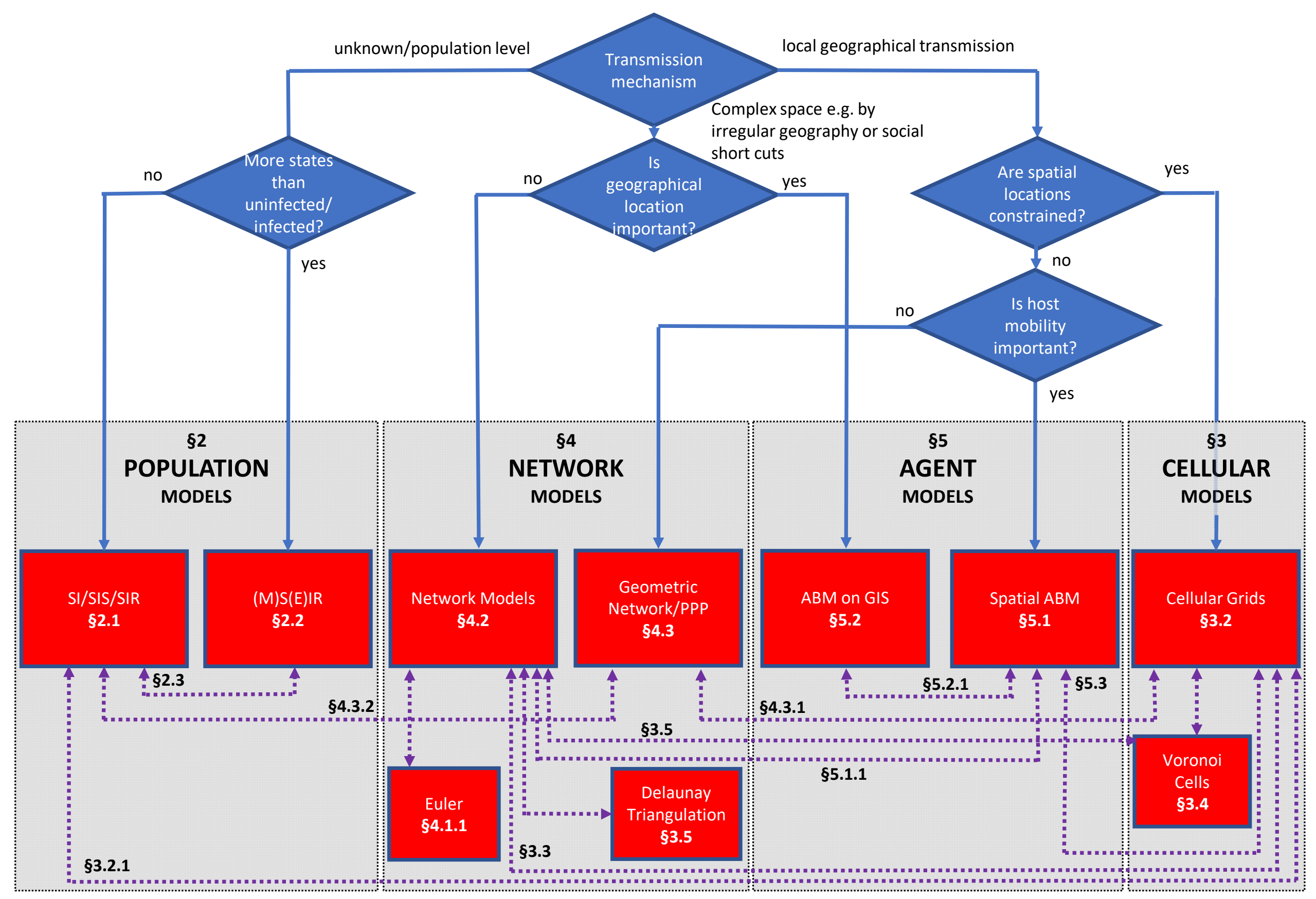

Figure 17: Spatial Transmission Modelling Flowchart showing Links (Dotted Purple Lines) Between Modelling Techniques 


\section{CONCLUSION}

The choice of modeling technique is often not one that is thought about with much deliberation - it may be a matter of 'the way we do things around here'. In this paper, we have set out a framework for determining not only which is an appropriate method, but also identifying relationships between apparently disparate methods. Modelling is not an exact science - the choice of modelling technique is a matter of experience, the availability of data, and the type of system that is being modelled.

We have presented a framework that guides the reader to an appropriate modelling technique for the particular spatial transmission phenomenon under investigation. It should be noted that a variety of modelling techniques can produce the same qualitative outcome - any of the models presented can, for instance, replicate logistic growth.

Furthermore, individual modelling techniques have for too long been considered in isolation, without allowing the analytical technique used for the analysis of a problem to change from one modelling technique to another. We have presented links between modelling techniques in order that modelers using one technique can explore different methodologies, whether of higher or lower complexity.

While the decision of how to model should not be taken away from the domain-specific expert, the introduction of a framework for deciding which techniques are possible, is a step towards producing more valid models, which is the ultimate goal, for practitioners and modelers alike.

Future research may combine network and analytical models with heterogeneous agents, combining network and agent-based approaches, to generate dynamic models of transmission. Without explicitly modelling the spatial component, by situating transmission agents in geographical space, risk analysis models may lead to erroneous policy interventions and costs to individuals, companies, and society as a whole. 


\section{REFERENCES}

Anderson, R. M. and May, R. M. (1979) 'Population Biology of Infectious Diseases: Part I', Nature, 280, 361-367

Anderson, R. M. and May, R. M. (1992) Infectious Diseases of Humans: Dynamics and Control, Oxford: Oxford University Press

Andrews, J. G., Ganti, R. K., Haenggi, M., Jindal, N., and Weber, S. (2010) 'A Primer on Spatial Modelling and Analysis in Wireless Networks', IEEE Communications Magazine, 48(11), 156-163

Atkinson, M. P., Cao, Z., Wein, L. M. (2008) 'Optimal Stopping Analysis of a Radiation Detection System to Protect Cities from a Nuclear Terrorist Attack', Risk Analysis, 28(2), 353-371

Axtell, R., Axelrod, R., Epstein, J. M., and Cohen, M. D. (1996) ‘Aligning Simulation Models: A Case Study and Results', Computational and Mathematical Organization Theory, 1, 123-141

Bak, P., Tang, C. and Wiesenfeld, K. (1987) 'Self-Organized Criticality: An Explanation of 1/f Noise', Physical Review Letters, 59 (4), 381-384

Bak, P., Chen, K., and Tang, C. (1990) 'A forest-fire model and some thoughts on turbulence', Phys. Lett. A, 147, 297-300

Bancal, J.-D. and Pastor-Satorras, P. (2010) 'Steady-State Dynamics of the Forest Fire Model on Complex Networks', European Physical Journal B, 76(1), 109-121

Barabási, A. L. (2002) Linked: The New Science of Networks, Cambridge MA: Perseus Books Barabási, A. L. and Albert, R. (1999) 'Emergence of Scaling in Random Networks', Science, 286, $509-512$

Barthélemy, M. (2011) ‘Spatial Networks’, Physics Reports, 499(1), 1-101

Batty, M., Couclelis, H., and Eichen, M. (1997) 'Urban Systems as Cellular Automata', Environment and Planning B: Planning and Design, 24, 159-164 
Berger, T. (2001) 'Agent-Based Spatial Models Applied to Agriculture: A Simulation Tool for Technology Diffusion, Resource Use Changes and Policy Analysis', Agricultural Economics, 25, $245-260$

Bradlow, E. T., Bronnenberg, B., Russell, G. J., Arora, N., Bell, D. R., Duvvuri, S. D., Hofstede, F. T., Sismeiro, C., Thomadsen, R., and Yang, S. (2005) 'Spatial Models in Marketing', Marketing Letters, 16(3/4), 267-278

Boccara, N. and Cheong, K. (1993) 'Critical Behavior of a Probabilistic Automata Network SIS Model for the Spread of an Infectious Disease in a Population of Moving Individuals', J. Phys. A: Math. Gen., 26, 3707-3717

Borshchev, A. (2013) 'Multi-Method Modeling', Proceedings of the 2013 Winter Simulation Conference, Pasupathy, R., Kim, S.-H., Tolk, A., Hill, R., and M. E. Kulh (Eds.), IEEE Publishing Brennan, A., Chick, S. E., and Davies, R. (2006) 'A Taxonomy of Model Structures for Economic Evaluation of Health Technologies', Health Economics, 15, 1295-1310

Broadbent, S. and Hammersley, J. (1957) 'Percolation Processes I: Crystals and Mazes', Proceedings of the Cambridge Philosophical Society, 53, 629-641

Brown, D. G., Riolo, R., Robinson, D. T., North, M., and Rand, W. (2005) 'Spatial Process and Data Models: Toward Integration of Agent-Based Models and GIS', Journal of Geographical Systems, $7(1), 25-47$

Carley, K. M., Fridsma, D., Casman, E., Yahja, A., Altman, N., Chen, L.-C., Kaminsky, B., and Nave, D. (2006) 'BioWar: Scaleable Agent-Based Model of Bioattacks', IEEE Transactions on Systems, Man, and Cybernetics, Part B (Cybernetics), 36(2), 252-265

Chen, K., Bak, P., and Jensen, M. H. (1990), 'A deterministic critical forest-fire model', Phys. Lett. A, $149,207-210$ 
Clarke, K. C. (2013) 'Cellular Automata and Agent-Based Models', Handbook of Regional Science, $1217-1233$

Colizza, V., Barrat, A., Barthélemy, M., and Vespignani, A. (2006) ‘The Role of Airline Transportation Network Prediction and Predictability of Global Epidemics', Proceedings of the National Academy of Sciences of the United States of America, 103(7), 2015-2020

Cox, L. A. (1999) 'Adaptive Spatial Sampling of Contaminated Soil', Risk Analysis, 19(6), 1059-1069

Crooks, A. T. and Wise, S. (2013) 'GIS and Agent-Based Models for Humanitarian Assistance', Computers, Environment and Urban Systems, 41, 100-111

Cross, P. C., Lloyd-Smith, J. O., Johnson, P. L. F., and Getz, W. M. (2005) 'Duelling Timescales of Host Movement and Disease Recovery Determine Invasion of Disease in Structured Populations', Ecology Letters, 8(6), 587-595

Delaunay, B. (1934) 'Sur la sphère vide’, Bulletin de l'Académie des Sciences de l'URSS, Classe des Sciences Mathématiques et Naturelles, 6, 793-800

Dettmann, C. P. and Georgiou, O. (2016) 'Random Geometric Graphs with General Connection Functions', Physical Review E, 93, 032313, 1-14

Drossel, B. and Schwabl, F. (1992), 'Self-organized critical forest-fire model', Phys. Rev. Lett., 69, $1629-1632$

Eisenberg, J. N., Seto, E. Y. W., Olivieri, A. W., and Spear, R. C. (1996) 'Quantifying Water Pathogen Risk in an Epidemiological Framework', Risk Analysis, 16(4), 549-563

Erdős, P. and Rényi, A. (1959) ‘On Random Graphs. I’, Publicationes Mathematicae, 6: 290-297

Erol, K., Levy, R., and Wentworth, J. (2000) 'Application of Agent Technology to Traffic Simulation', US Department of Transportation 
Eubank, S., Guclu, H., Kumar, V. S. A., Marathe, M. V., Srinivasan, A., Toroczkai, Z., Wang, N. (2004) 'Modelling Disease Outbreaks in Realistic Urban Social Networks, Nature, 429, 180-184

Euler, L. (1736) 'Solutio Problematis ad Geometriam Situs Pertinentis', Commentarii Academiae Scientiarum Imperialis Petropolitanae, 8, 128-140

Fann, N., Lamson, A. D., Anenberg, S. C., Wesson, K., Risley, D., and Hubbell, B. J. (2012) 'Estimating the National Public Health Burden Associated with Exposure to Ambient $\mathrm{PM}_{2.5}$ and Ozone', Risk Analysis, 32(1), 81-95

Frosch, R. A. (2004) 'Notes Towards a Theory of Accident Precursors and Catastrophic System Failure', In: Phimister, J. R., Bier, V. M., Kunreuther, H. C. (Eds.) Accident Precursor Analysis and Management: Reducing Technological Risk Through Diligence, National Academy of Engineering of the National Academies, Washington DC: National Academies Press, pp177-181

Gastner, M. T. and Newman, E. J. (2004) 'Diffusion-Based Method for Producing Density-Equalizing Maps', Proceedings of the National Academy of Sciences of the United States of America, 101(20), $7499-7504$

Gastner, M. T. and Newman, E. J. (2006) 'The Spatial Structure of Networks', European Physical Journal B, 49, 247-252

Gebetsroither-Geringer, E. (2014) 'Multimethod Modeling and Simulation Supporting Urban Planning Decisions', Understanding Complex Urban Systems: Multidisciplinary Approaches to Modeling, Walloth, C., Gurr, J. M., Schmidt, J. A. (Eds.), Springer

Gilbert, E.N. (1959) 'Random Graphs’, Annals of Mathematical Statistics, 30 (4), 1141-1144

Gilbert, E. N. (1961) 'Random Plane Networks', J. Soc. Indust. Appl. Math., 9(4), 533-543

Golstein, J. (1999) 'Emergence as a Construct: History and Issues', Emergence, 1(1), 49-72

González, M. C., Lind, P. G., and Herrmann, H. J. (2006) 'Model of Mobile Agents for Sexual Interactions Networks', European Physical Journal B, 49, 371-376 
Helal, M., Rabelo, L., Sepúlveda, J. and Jones, A. (2007) ‘A Methodology for Integrating and Synchronizing the System Dynamics and Discrete Event Simulation Paradigms' In: Proceedings of the 25th International Conference of the System Dynamics Society, J. D. Sterman, M. P. Repenning, R. S. Langer, J. I. Rowe, and J. M. Yarni (Eds.), 3:1-24. Boston, MA: System Dynamics Society Heppenstall, A. J., Crooks, A. T., See, L. M., and Batty, M. (Eds.) Agent-Based Models of Geographical Systems, Heidelberg: Springer

Hethcote, H.W . (2000) 'The Mathematics of Infectious Diseases', SIAM Review, 42(4), 599-653

Holland, J.H. and Miller, J.H. (1991) ‘Artificial Adaptive Agents in Economic Theory', American Economic Review, 81(2), 365-71

Isham, V., Kaczmarska, J., and Nekovee, M. (2011) 'Spread of Information and Infection on Finite Random Networks', Physical Review E, 83, 046128, 1-12

Jespersen, S. and Blumen, A. (2000) 'Small-World Networks: Links with long-tailed distributions', Physical Review E, 62, 6270

Kaiser, M. and Hilgetag, C. C. (2004) 'Spatial Growth of Real-World Networks', Physical Review E, 69, 036103, 1-5

Karinthy, F. (1929) L'aancszemek (Chains)

Kasperson, R. E., Renn, O., Slovic, P., Brown, H. S., Emel, J., Goble, R., Kasperson, J.X., and Ratick, S. (1988) 'The Social Amplification of Risk: A Conceptual Framework', Risk Analysis, 8(2), 177-187

Kermack, W. O., and McKendrick, A. G. (1927) 'A Contribution to the Mathematical Theory of Epidemics', Proceedings of the Royal Society A, 115(772), 700-721

Kleinberg, J. M. (2000) 'Navigation in a Small World', Nature, 406, 845

Koch, T. (2004) 'The Map as Intent: Variations on the Theme of John Snow', Cartographica, 39(4), $1-14$ 
Lloyd-Smith, J. O., Schreiber, S. J., Kopp, P.E., and Getz, W. M. (2005) 'Superspreading and the Effect of Individual Variation on Disease Emergence', Nature, 438, 355-359

Milgram, S. (1967) ‘The Small-World Problem', Psychology Today, 1(1), 61-67

Mollison, D. and Kuulasmaa, K. (1985) ‘Spatial Epidemic Models: Theory and Simulation’, In: Bacon, P. (Ed.), Population Dynamics of Rabies in Wildlife. Academic Press, London; Orlando, pp. $291-309$

Morabia, A. (2004) A History of Epidemiologic Methods and Concepts, Basel: Birkhäuser Verlag

Morecroft, J. D. W. and Robinson, S. (2005) 'Explaining Puzzling Dynamics: Comparing the Use of System Dynamics and Discrete-Event Simulation', Proceedings of the $23^{\text {rd }}$ Conference of the System Dynamics Society, Sterman, J. D., Repenning, N. P., Langer, R. S., Rowe, J. I., and Yanni, J. M. (Eds.)

Moreno, J. and Alvar, J. (2002) 'Canine Leishmaniasis: Epidemiological Risk and the Experimental Model', Trends in Parasitology, 18(9), 399-405

Merian, M. and Merian, C. (1652) 'Koningsberga', Theatrum Europaenum, Merian Erben, Basel Nekovee, M. (2007) 'Worm Epidemics in Wireless Ad Hoc Networks', New Journal of Physics, 9, 189

Nair, A. and Vidal, J. M. (2011) 'Supply Network Topology and Robustness Against Disruptions An Investigation using Multi-Agent Model', International Journal of Production Research, 49(5), $1391-1404$

North, M. J., Collier, N., Ozik, J., Tatara, E. R., Macal, C. M., Bragen, M., and Sydelko, P. (2013)

'Complex Adaptive Systems Modeling with RePast Symphony', Complex Adaptive Systems Modeling, 1(3), 1-26

Pfeiffer, D. U., Robinson, T. P., Stevenson, M., Stevens, K. B., Rogers, D. J., and Clements, A. C. A., Spatial Analysis in Epidemiology, Oxford: Oxford University Press 
Rahmandad, H. and Sterman, J. (2008) 'Heterogeneity and Network Structure in the Dynamics of Diffusion: Comparing Agent-Based and Differential Equation Models', Management Science, 54(5), 998-1014

Riley, S. (2007) 'Large-Scale Spatial-Transmission Models of Infectious Disease’, Science, 316(5829), 1298-1301

Riley, S., Eames, K., Isham, V., Mollison, D., and Trapman, P. (2015) 'Five Challenges for Spatial Epidemic Models', Epidemics, 10(1), 68-71

Robertson, D. A. (2003) 'Agent-Based Models of a Banking Network as an Example of a Turbulent Environment: The Deliberate vs. Emergent Strategy Debate Revisited', Emergence, 5(2), 56-71

Robertson, D. A. (2005) ‘Agent-Based Modeling Toolkits: NetLogo, RePast, and Swarm’, Academy of Management Learning and Education, 4(4):524-527

Robertson, D. A. and Caldart, A. A. (2009) The Dynamics of Strategy: Mastering Strategic Landscapes of the Firm, Oxford: Oxford University Press

Schelling, T. C. (1971) 'Dynamic Models of Segregation', Journal of Mathematical Sociology, 1, $143-186$

Schelling, T. C. (1978) Micromotives and Macrobehaviors, New York; London: Norton

Sen, P. and Chakrabarti, B. K. (2001) 'Small-World Phenomena and the Statistics of Linear Polymers', Journal of Physics A, 34, 7749-7755

Shanthikumar, J. G. and Sargent, R. G. (1983) ‘A Unifying View of Hybrid Simulation/Analytic Models and Modeling', Operations Research, 31(6), 1030-1052

Shi, W. and Pang, M. Y. C. (2000) 'Development of Voronoi-based Cellular Automata - An Integrated Dynamic Model for Geospatial Information Systems', Int. J. Geographical Information Science, 14(5), 455-474 
Sikder, I. U., Mal-Sarkar, S., and Mal, T. K. (2006) 'Knowledge-Based Risk Assessment Under Uncertainty for Species Invasion', Risk Analysis, 26(1), 239-252

Sloot, P. M. A., Ivanov, S. V., Boukhanovsky, A. V., van de Vijver, D. A. M. C., and Boucher, C. A. B. (2008) 'Stochastic Simulation of HIV Population Dynamics through Complex Network Modelling', International Journal of Computer Mathematics, 85(8), 1175-1187

Snow J. (1855) On the Mode of Communication of Cholera, 2nd ed. London: Churchill

Stoddard, S. T., Morrison, A. C., Vazquez-Prokopec, G. M., Soldan, V. P., Kochel, T. J., Kitron, U., Elder, J. P., and Scott, T. W. (2009) 'The Role of Human Movement in the Transmission of VectorBorne Pathogens', PLOS Neglected Tropical Diseases, 3(7), 1-9

Stoneham, A. K. M. (1977) 'The Small World Problem in a Spatial Context', Environment and Planning A, 9, 185-195

Tekippe, A. J. and Krejci, C. C. (2016) 'Using Hybrid Simulation to Assess the Dynamics of Compassion Fatigue in Veterinarian General Practitioners', In: Proceedings of the 2016 Winter Simulation Conference, T. M. K. Roeder, P. I. Frazier, R. Szechtman, E. Zhou, T. Huschka, and S. E. Chick (Eds.), IEEE Press

Thompson, K. M. (2016) 'Evolution of Use of Dynamic Transmission Models for Measles and Rubella Risk and Policy Analysis', Risk Analysis, 36(7), 1383-1403

Rothermel, R. C. (1972) ‘A Mathematical Model for Predicting Fire Spread in Wildland Fuels', USDA Forest Service Research Paper INT-115

Torrens, P. M. and Benenson, I. (2005) ‘Geographic Automata Systems’, International Journal of Geographical Information Science, 19(4), 385-412

Turner, M. G. (1987) 'Spatial Simulation of Landscape Changes in Georgia: A Comparison of three Transition Models', Landscape Ecology, 1(1), 29-36 
Watts, D. J. and Strogatz, S. H. (1998) 'Collective Dynamics of “Small-World” Networks', Nature, 393(6684), 440-442

White, S. H., del Rey, A. M., Sanchez, G. R. (2007) 'Modeling Epidemics using Cellular Automata', Applied Mathematics and Computation, 186(1), 193-202

Wilensky, U. (2006) 'NetLogo Voronoi Model', Center for Connected Learning and Computer-Based Modeling, Northwestern University, Evanston IL

Wilensky, U. (2017) 'NetLogo GIS Gradient Example', Center for Connected Learning and Computer-Based Modeling, Northwestern University, Evanston IL

With, K. A. (2004) 'Assessing the Risk of Invasive Spread in Fragmented Landscapes', Risk Analysis, 24(4), 803-815

With, K. A. and King, A. W. (1997) 'The Use and Abuse of Neutral Landscape Models in Ecology', Oikos, 79(2), 219-229

Zagmutt, F. J., Schoenbaum, M. A., and Hill, A. E. (2016) 'The Impact of Population, Contact, and Spatial Heterogeneity on Epidemic Model Predictions', Risk Analysis, 36(5), 939-953

Zhou, Y. (2015) 'Tutorial on Using and Exporting GIS Vector Dataset in NetLogo', http://geospatialcss.blogspot.co.uk/2015/10/tutorial-on-using-and-exporting-gis.html, accessed 21 February 2018

Ziff, R. M. (2009) 'Explosive Growth in Biased Dynamic Percolation on Two-Dimensional Regular Lattice Networks', Physical Review Letters, 103, 045701

Zio, E. and Sansavini, G. (2011) 'Component Criticality in Failure Cascade Processes of Network Systems', Risk Analysis, 31(8), 1196-1210 